\title{
Pericyte-Mediated Tissue Repair through PDGFR $\beta$ Promotes Peri-Infarct Astrogliosis, Oligodendrogenesis, and Functional Recovery after Acute Ischemic Stroke
}

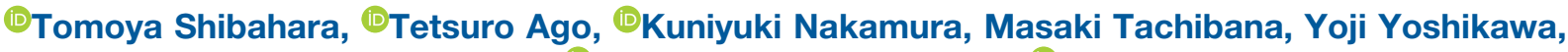
Motohiro Komori, Kei Yamanaka, (1)Yoshinobu Wakisaka, and (DTakanari Kitazono

https://doi.org/10.1523/ENEURO.0474-19.2020

Department of Medicine and Clinical Science, Graduate School of Medical Sciences, Kyushu University, Fukuoka 8128582, Japan

\section{Visual Abstract}

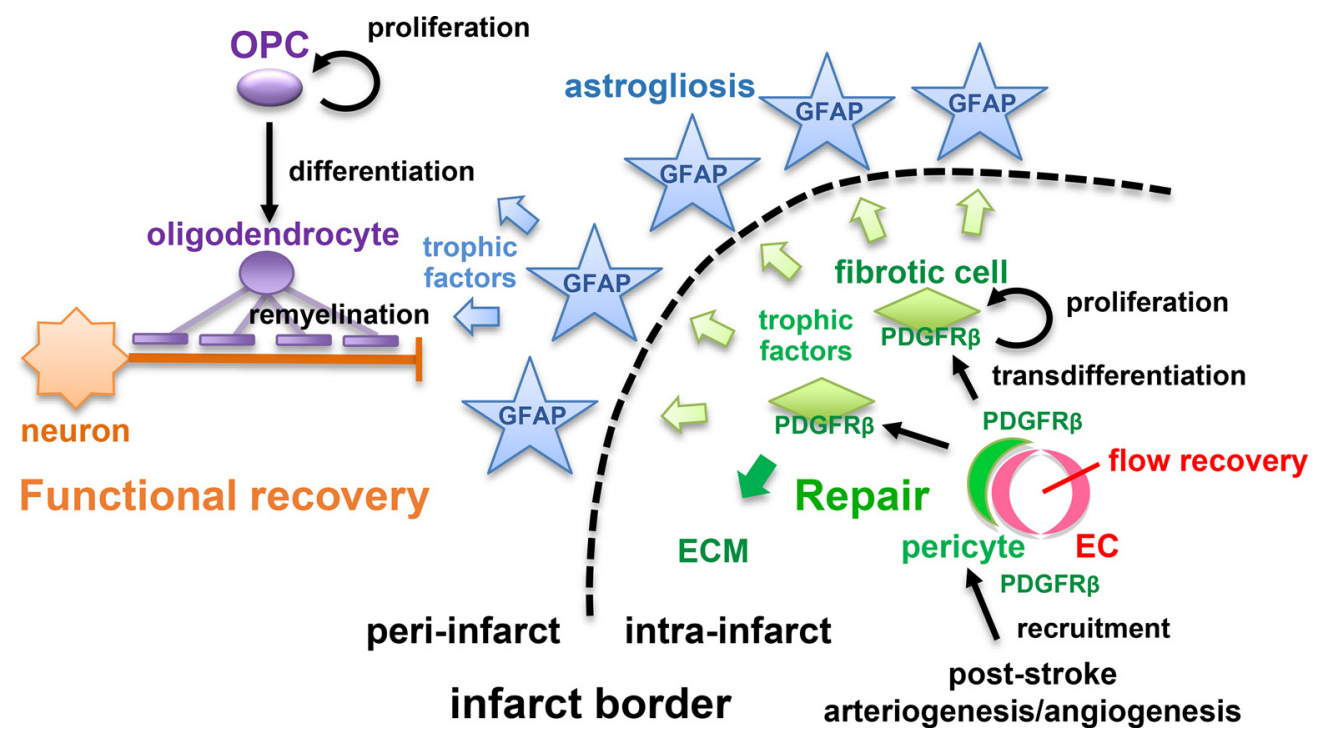

\section{Significance Statement}

Pericyte-mediated fibrotic tissue repair is a major histological change within the infarct area during the subacute phase after ischemic stroke. Whether fibrotic repair is beneficial or detrimental to post-stroke functional recovery is highly debated. Here, we demonstrate that inhibition of fibrotic repair in mice by heterozygous deletion of platelet-derived growth factor receptor $\beta$ (PDGFR $\beta)\left(\right.$ Pdgfrb $\left.^{+/-}\right)$significantly attenuates functional recovery after ischemic stroke. Pericyte-derived PDGFR $\beta$-positive cells within the infarct area produced trophic factors that activated astrocytes, thereby enhancing peri-infarct astrogliosis. Furthermore, astrocytes, conditioned with PDGF-BB-stimulated pericyte culture medium, promoted oligodendrocyte $(\mathrm{OL})$ differentiation and a myelinating response. Peri-infarct oligodendrogenesis and re-myelination within areas of astrogliosis was significantly attenuated in $\mathrm{Pdgfrb}^{+/-}$mice. Pericyte-mediated tissue repair is beneficial for post-stroke functional recovery and is a potential therapeutic target. 
Post-stroke functional recovery can occur spontaneously during the subacute phase; however, how poststroke fibrotic repair affects functional recovery is highly debated. Platelet-derived growth factor receptor $\beta$ (PDGFR $\beta$ )-expressing pericytes are responsible for post-stroke fibrotic repair within infarct areas; therefore, we examined peri-infarct neural reorganization and functional recovery after permanent middle cerebral artery occlusion (pMCAO) using pericyte-deficient $\mathrm{Pdgfrb}^{+/-}$mice. Time-dependent reduction of infarct area sizes, i.e., repair, was significantly impaired in $\mathrm{Pdgfrb}^{+/-}$mice with recovery of cerebral blood flow (CBF) in ischemic areas attenuated by defective leptomeningeal arteriogenesis and intrainfarct angiogenesis. Peri-infarct astrogliosis, accompanied by increased STAT3 phosphorylation, was attenuated in Pdgfrb ${ }^{+/-}$mice. Pericyte-conditioned medium (PCM), particularly when treated with platelet-derived growth factor subunit $B$ (PDGFB) homodimer (PDGF-BB; PCM/PDGF-BB), activated STAT3 and enhanced the proliferation and activity of cultured astrocytes. Although peri-infarct proliferation of oligodendrocyte (OL) precursor cells (OPCs) was induced promptly after pMCAO regardless of intrainfarct repair, OPC differentiation and remyelination were significantly attenuated in Pdgfrb ${ }^{+/}$mice. Consistently, astrocyte-CM (ACM) promoted OPC differentiation and myelination, which were enhanced remarkably by adding PCM/PDGF-BB to the medium. Post-stroke functional recovery correlated well with the extent and process of intrainfarct repair and peri-infarct oligodendrogenesis. Overall, pericyte-mediated intrainfarct fibrotic repair through PDGFR $\beta$ may promote functional recovery through enhancement of peri-infarct oligodendrogenesis as well as astrogliosis after acute ischemic stroke.

Key words: astrocyte; neurorestoration; oligodendrogenesis; pericyte; platelet-derived growth factor receptor $\beta$; repair

\section{Introduction}

Stroke is a leading cause of death and disability worldwide. Extensive efforts have been made to explore neuroprotective and neurorestorative therapies against acute ischemic stroke. However, most clinical trials targeting acute phase neuroprotection, based on promising results in animal experiments, have ended in failure, largely because of the limited therapeutic time window (Ginsberg, 2008; Xing et al., 2017). Exceptions include reperfusion therapies, either by intravenous infusion of recombinant tissue plasminogen activator (National Institute of Neurological D and Stroke rt-PA Stroke Study Group, 1995) or by catheter-mediated thrombectomy (Goyal et al., 2016). To achieve neuroprotection, focus should be paid to roles of non-neuronal components in

Received November 16, 2019; accepted January 23, 2020; First published February 5, 2020.

The authors declare no competing financial interests.

Author contributions: T.S., T.A., K.N., Y.W., and T.K. designed research; T.S., T.A., M.T., Y.Y., M.K., K.Y., and Y.W. performed research; T.S., T.A., K.N., M.T., Y.Y., M.K., K.Y., and Y.W. analyzed data; T.S., T.A., K.N., Y.W., and T.K. wrote the paper.

This work was supported by Grants-in-Aid for Scientific Research (B) $16 \mathrm{H} 05439$ (to T.A. and T.K.), (C) 26462163 (GAG9K09530) (to Y.W.), and (C) GAG9K09511 (to K.N.) from the Ministry of Education, Culture, Sports, Science and Technology, Japan; a grant from SENSHIN Medical Research Foundation, Japan (T.A. and K.N.); and research grants from Astellas, Boehringer Ingelheim, Bristol-Myers Squibb, Daiichi Sankyo, Eisai, MSD, and Takeda (T.A. and T.K.).

Acknowledgments: We thank Jeremy Allen, PhD, from Edanz Group for editing a draft of this manuscript. We also thank Hideko Noguchi (Kyushu University) and Naoko Kasahara (Hisayama Research Institute for Lifestyle Diseases) for technical support.

Correspondence should be addressed to Tetsuro Ago at agou@intmed2. med.kyushu-u.ac.jp.

https://doi.org/10.1523/ENEURO.0474-19.2020

Copyright @ 2020 Shibahara et al.

This is an open-access article distributed under the terms of the Creative Commons Attribution 4.0 International license, which permits unrestricted use, distribution and reproduction in any medium provided that the original work is properly attributed. the brain, such as vascular cells and glial cells, i.e., the concept of the neurovascular unit (Lo et al., 2003). We should also note that post-stroke functional recovery can occur spontaneously for up to three to six months with the aid of rehabilitation therapy (Cramer, 2008). The extent of recovery is often different between individuals, even when infarcts are of similar size and localization. Epidemiological studies also demonstrate that various factors, including age, sex, and status of life-style diseases, can affect post-stroke functional recovery. These findings encourage the exploration of therapeutic strategies that promote recovery of function, i.e., neurorestoration, even during the subacute phase after ischemic stroke (Chen et al., 2014; Tachibana et al., 2017; Xing et al., 2017).

Neurogenesis, astrogliosis, and oligodendrogenesis can occur in peri-infarct areas during the subacute phase. Although post-stroke neurogenesis has been extensively studied as a promising strategy for functional recovery, neural stem cells (NSCs) migrating from the subventricular zone (SVZ) into peri-infarct areas mostly differentiate into reactive astrocytes and contribute to astrogliosis, despite the NSCs having the potential to differentiate into neurons (Ohab et al., 2006; Faiz et al., 2015). It was believed that peri-infarct astrogliosis formed by migrating NSCs and resident astrocytes hindered axonal regrowth and impaired functional recovery; however, accumulating evidence demonstrates that astrogliosis may not simply isolate necrotic from healthy tissue to prevent the spread of damage, but that it may aid axonal regrowth and remyelination after ischemic stroke (Zamanian et al., 2012; Faiz et al., 2015; Anderson et al., 2016). In addition to astrogliosis, oligodendrogenesis is now recognized as a promising therapeutic target to promote functional recovery, not only in demyelinating disorders (Tognatta and Miller, 2016) but also in ischemic stroke (Iwai et al., 2010; Zhang et al., 2013; Itoh et al., 2015). Oligodendrocyte (OL) precursor cells (OPCs) make up $5 \%$ of the glial population 
and are diffusely distributed in the normal adult brain. Locally pre-existing OPCs, in addition to OPCs migrating from the SVZ, can differentiate into OLs to accomplish remyelination at sites of brain injury with demyelination, including the so-called penumbra (Levine et al., 2001; Itoh et al., 2015; Rosenzweig and Carmichael, 2015). Remyelination can restore neuronal functions and also prevent axonal degeneration leading to neuronal death; therefore, post-stroke enhancement of oligodendrogenesis is a promising strategy for functional recovery.

In addition to the peri-infarct neural reorganization, intrainfarct fibrosis is a prominent pathological change, in which pericytes play a pivotal role, during the subacute phase after ischemic stroke. Pericytes are vascular mural cells interacting directly with endothelial cells in microvessels at a high ratio in the CNS (Armulik et al., 2005). They regulate angiogenesis, stability of the blood-brain barrier (BBB) formed by endothelial cells, and cerebral blood flow (CBF) through the actions of platelet-derived growth factor receptor $\beta$ (PDGFR $\beta$; Daneman et al., 2010; Armulik et al., 2011; Sweeney et al., 2016). Furthermore, pericyte-derived PDGFR $\beta$-expressing cells function as fibrosis-forming cells after ischemic stroke (Göritz et al., 2011; Makihara et al., 2015). Although a difficulty of pericyte research in the CNS is the lack of pericyte-specific markers, some reports have demonstrated that PDGFR $\beta$ can be used as a pericyte marker, particularly after ischemic stroke: the expression of PDGFR $\beta$ is induced remarkably and exclusively in pericytes within infarct areas (Winkler et al., 2010; Arimura et al., 2012). In these situations, it is still controversial whether pericyte-mediated fibrotic repair is beneficial or detrimental to peri-infarct neural reorganization and functional recovery (Su et al., 2008; Göritz et al., 2011; Makihara et al., 2015; Tachibana et al., 2017; Dias et al., 2018; Gautam and Yao, 2018). Thus, this is an important issue to be solved even from the standpoint of the development of novel therapeutic strategies that promote post-stroke functional recovery. In the present study, using heterozygous Pdgfrb knock-out $\left(P d g f r b^{+/-}\right)$mice, we aimed to elucidate how pericytemediated tissue repair within infarct areas affects peri-infarct neural reorganization and whether it can lead to better functional recovery after acute ischemic stroke.

\section{Materials and Methods}

\section{Animals}

Animal experiments were conducted according to the Guidelines for Proper Conduct of Animal Experiments by the National Science Council of Japan. The Animal Care and Use Review Committee of the Kyushu university approved our animal procedures. 129S Pdgfrb ${ }^{+/}$mice were purchased from The Jackson Laboratory (https://www.jax. org/strain/007846). 129S Pdgfrb ${ }^{+/}$mice were backcrossed with C57BL/6 ten times to produce C57BL/6 Pdgfrb $^{+/-}$ mice. Homozygous deletion of Pdgfrb (Pdgfrb $\left.{ }^{-\prime}\right)$ is embryonic lethal (Soriano, 1994); therefore, we used Pdgfrb ${ }^{+/}$ mice to characterize the roles of PDGFR $\beta$ after ischemic stroke. We used mainly male mice (and female mice when indicated) aged 8-15 weeks and weighing 20-30 g. Mice were bred and housed two per cage in the authors' animal facility at $21^{\circ} \mathrm{C}$ and $65 \%$ humidity with a regulated $12 / 12 \mathrm{~h}$ light/dark cycle and with free access to food and water. All experiments were reported according to the ARRIVE guidelines.

\section{Blood pressure (BP) and heart rate (HR) measurement}

Mouse BP and HR were measured using the non-invasive tail-cuff BP system (BP-2000, Visitech Systems). Briefly, mice were trained with 10 consecutive preliminary measurements, followed by 25 study measurements that were averaged for each individual animal. The procedure was considered successful when 20 out of the 25 measurements were valid. Mice were conditioned to the BP monitoring procedure for three consecutive days before experiments. At day 0 before pMCAO, the baseline BP was measured. BP was measured again at days 1, 3, 7, and 14 after pMCAO.

\section{Mouse stroke model}

Mice were randomly assigned to the animal surgeon and were anesthetized by the inhalation of $2 \%$ isoflurane in air and maintained under anesthesia with $1.5 \%$ isoflurane. Rectal temperatures were maintained at $35-37^{\circ} \mathrm{C}$ with a heat lamp. CBF before and during ischemia was measured at the ipsilateral parietal bone $(2 \mathrm{~mm}$ posterior and $4 \mathrm{~mm}$ lateral to bregma) using a laser Doppler flowmeter (PeriFlux System 5000, PERIMED). Focal cerebral ischemia was induced by permanent occlusion of the right middle cerebral artery (pMCAO) using a laser-induced photochemical reaction as described previously (Makihara et al., 2015). Briefly, the right jugular vein was exposed and a catheter was inserted into the superior vena cava for intravenous administration of a solution. After the right distal MCA was carefully exposed, a diodepumped solid-state laser was used to irradiate the distal MCA at a wavelength of $561 \mathrm{~nm}$ with $6 \mathrm{~mW}$ of emitted power. Upon laser irradiation, a photosensitizing Rose bengal dye solution (20 mg/kg, Wako \#184-00272) was administrated intravenously for $90 \mathrm{~s}$. After $4 \mathrm{~min}$ of irradiation, the laser beam was refocused on the MCA just proximal to the first position, followed by another 4 min of irradiation. Then, the right common carotid artery was ligated with a $6-0$ silk suture.

\section{Measurement of CBF by laser speckle flowmetry}

Relative CBF was determined by laser speckle flowmetry, which obtains high-resolution 2D imaging (OMEGAZONE OZ-2, OMEGAWAVE, INC.) and has a linear relationship with absolute CBF values (Ayata et al., 2004). Recordings were performed through the skull under anesthesia with $1.5 \%$ isoflurane. The majority of the periosteum, which adheres to the skull, was removed with fine-tip forceps. For each recording, the skull surface was wiped with salinesoaked gauze. The mean CBF of the ischemic core, located $2 \mathrm{~mm}$ posterior and $4 \mathrm{~mm}$ lateral to bregma, was measured in identically sized regions of interest (ROls; 900 pixels). The absolute CBF value is theoretically vulnerable to many experimental conditions, such as magnification of the charge- 
coupled device camera and room light, so it may not always be reliable (Ayata et al., 2004). Therefore, we used the relative $\mathrm{CBF}$ of an $\mathrm{ROI}$ expressed as a percentage of the CBF value of the ipsilateral $\mathrm{ROI}$ against that of the corresponding $\mathrm{ROI}$ in the contralateral hemisphere.

\section{Latex perfusion method to assess leptomeningeal collaterals}

In another set of mice, leptomeningeal collaterals were visualized $7 \mathrm{~d}$ after PMCAO. Under deep pentobarbital anesthesia, the right atrium of the heart was incised to allow for venous outflow. The left ventricle of the heart was cannulated, and 2-ml saline was injected. Immediately after the saline injection, 0.5-ml white latex compound (product no. 563; Chicago Latex Products Inc) mixed with $50 \mu \mathrm{l} / \mathrm{ml}$ carbon black (Bokusai; Fuekinori Kogyo Co, Ltd) was injected. The injection pressure was about $150 \mathrm{mmHg}$ as reported previously (Komori et al., 2019). The brains were fixed with $4 \%$ paraformaldehyde (PFA) in PBS for $2 \mathrm{~d}$. Photographs of the brain were taken at $50 \times$ magnification to measure the vessel diameter of the leptomeningeal anastomosis. The distal MCA was identified from its branch angle and distinguished from the distal ACA or posterior cerebral artery. The diameter of the leptomeningeal anastomosis was measured at the point of confluence between the distal MCA and the distal ACA. Independent persons not involved in the animal procedures performed the measurements.

\section{Assessment of BBB breakdown}

Permeability of the BBB in infarct areas on day 28 was assessed by the leakage of Evans blue dye (SigmaAldrich \#E2129). Briefly, the brain was collected 60 min after a $2 \%$ solution of Evans blue dye $(4 \mathrm{ml} / \mathrm{kg}$ body weight) was injected intravenously via the tail vein. After mice were euthanized and transcardially perfused with ice-cold saline, whole brains were fixed with 4\% PFA in PBS.

\section{Immunohistochemistry and immunofluorescence}

Mice were killed at the indicated day after pMCAO by intraperitoneal administration of pentobarbital (150 $\mathrm{mg} / \mathrm{kg}$ body weight) and transcardially perfused with $20-\mathrm{ml}$ ice-cold saline followed by $20-\mathrm{ml} 4 \%$ PFA in PBS at $4{ }^{\circ} \mathrm{C}$. Whole brains were fixed with $4 \%$ PFA in PBS for approximately $3 \mathrm{~d}$. PFA-fixed 2-mm-thick coronal slices were embedded in paraffin and sectioned at $4 \mu \mathrm{m}$. The sections were then deparaffinized, rehydrated through a graded series of ethanol solutions, and washed in PBS (Tachibana et al., 2017). After blocking with a solution of $5 \%$ skimmed milk or PBS containing $10 \%$ normal goat serum for $30 \mathrm{~min}$ at room temperature, the sections were incubated with primary antibodies: anti-microtubule-associated protein 2 (MAP2; 1:1000; Sigma-Aldrich \#M4403), antiPDGFR $\beta$ [1:100; Cell Signaling Technology (CST) \#3169], anti-CD13 (1:200; R\&D Systems \#AF2335), anti-CD31 (1:200; BD Biosciences \#550274), anti-glial fibrillary acidic protein (GFAP; 1:200; CST \#3670), anti-OLIG2 (1:200; R\&D
Systems \#AF2418), anti-APC (1:100; Calbiochem \#OP80), anti-ionized calcium-binding adapter molecule 1 (IBA1; 1:500; Abcam \#ab5076), anti-myelin basic protein (MBP; 1:500; Millipore \#MAB386), anti-SMI312 (1:500; BioLegend \#837904), anti-doublecortin (DCX; 1:1000; Millipore \#AB2253), anti-Fibrin (1:1000; DAKO \#A0080), anti-NG2 (1:200; Millipore \#AB5320), anti-interleukin-6 (IL6; 1:200; R\&D Systems \#AF206), or anti-pSTAT3 (1:1000; CST \#9145) at $4^{\circ} \mathrm{C}$ overnight. After washing with PBS/Triton X-100, the sections were incubated with appropriate secondary antibodies conjugated to Alexa Fluor dyes (Thermo Fisher Scientific) or stained with $3,3^{\prime}$-diaminobenzidine (DAB) using an appropriate kit (Nichirei). For DAB staining, endogenous peroxidase was inactivated with $0.3 \%$ hydrogen peroxide for 30 min before blocking with skimmed milk solution. The sections were observed on a BIOREVO BZ-9000 microscope (Keyence). The same conditions were used to stain and observe all sections. The same color tone in all sections was converted to grayscale and measured at the same threshold with ImageJ software. Independent persons not involved in animal procedures performed the image analysis. MAP2-negative areas, infarct volumes, and tissue atrophy were calculated using six sections spaced $400 \mu \mathrm{m}$ apart (from bregma $+1.4 \mathrm{~mm}$ to bregma $-0.6 \mathrm{~mm}$ ). The actual infarct volumes with corrections for edema were calculated as the volume of the contralateral hemisphere minus the non-infarcted volume of the ipsilateral hemisphere. Tissue atrophy was calculated as the MAP2-positive volume of the contralateral hemisphere minus that of the ipsilateral hemisphere (Shi et al., 2017). CD13-, CD31-, and Fibrin-positive areas were obtained using three sections spaced $400 \mu \mathrm{m}$ apart (from bregma $+1.0 \mathrm{~mm}$ to bregma $-0.2 \mathrm{~mm}$ ). Intrainfarct CD13- or CD31- positive areas $(\%)=(C D 13-$ or CD31- positive areas within infarct areas)/(infarct areas) $\times$ 100. OLIG2-, APC-, and IBA1-positive cells in the striatum were calculated using three sections spaced $400 \mu \mathrm{m}$ apart (from bregma $+1.0 \mathrm{~mm}$ to bregma $-0.2 \mathrm{~mm}$ ). To determine the density of positive cells, four randomly selected squares $(400 \times 400 \mu \mathrm{m})$ in the striatum were analyzed. We evaluated the MBP/ SMI312 ratio in the striatum in an area $100 \mu \mathrm{m}$ from the infarct area edge. The DCX-positive area in an area from the SVZ to the infarct was examined in five sections spaced $400 \mu \mathrm{m}$ apart (from bregma $+1.4 \mathrm{~mm}$ to bregma $-0.2 \mathrm{~mm}$ ). Peri-infarct GFAP-positive areas were calculated using three sections spaced $400 \mu \mathrm{m}$ apart (from bregma +1.0 $\mathrm{mm}$ to bregma $-0.2 \mathrm{~mm}$ ). To determine the density of positive cells, four randomly selected squares $(400 \times 400 \mu \mathrm{m})$ were analyzed. The number of GFAP and PSTAT3 doublepositive cells was evaluated from three serial sections spaced $400 \mu \mathrm{m}$ apart (from bregma $+1.0 \mathrm{~mm}$ to bregma $0.2 \mathrm{~mm}$ ) in the peri-infarct area on day 28. Six to eight animals per group were analyzed.

\section{Cortical whole mount immunostaining}

Mice were euthanized on day 7 by intraperitoneal administration of pentobarbital and transcardially perfused with $20-\mathrm{ml}$ ice-cold saline and $20-\mathrm{ml} 4 \%$ PFA. For immunostaining, the cortical surface of the brain was dissected (Okyere et al., 2018). Whole mounts were subsequently 
Table 1. Primers used for PCR

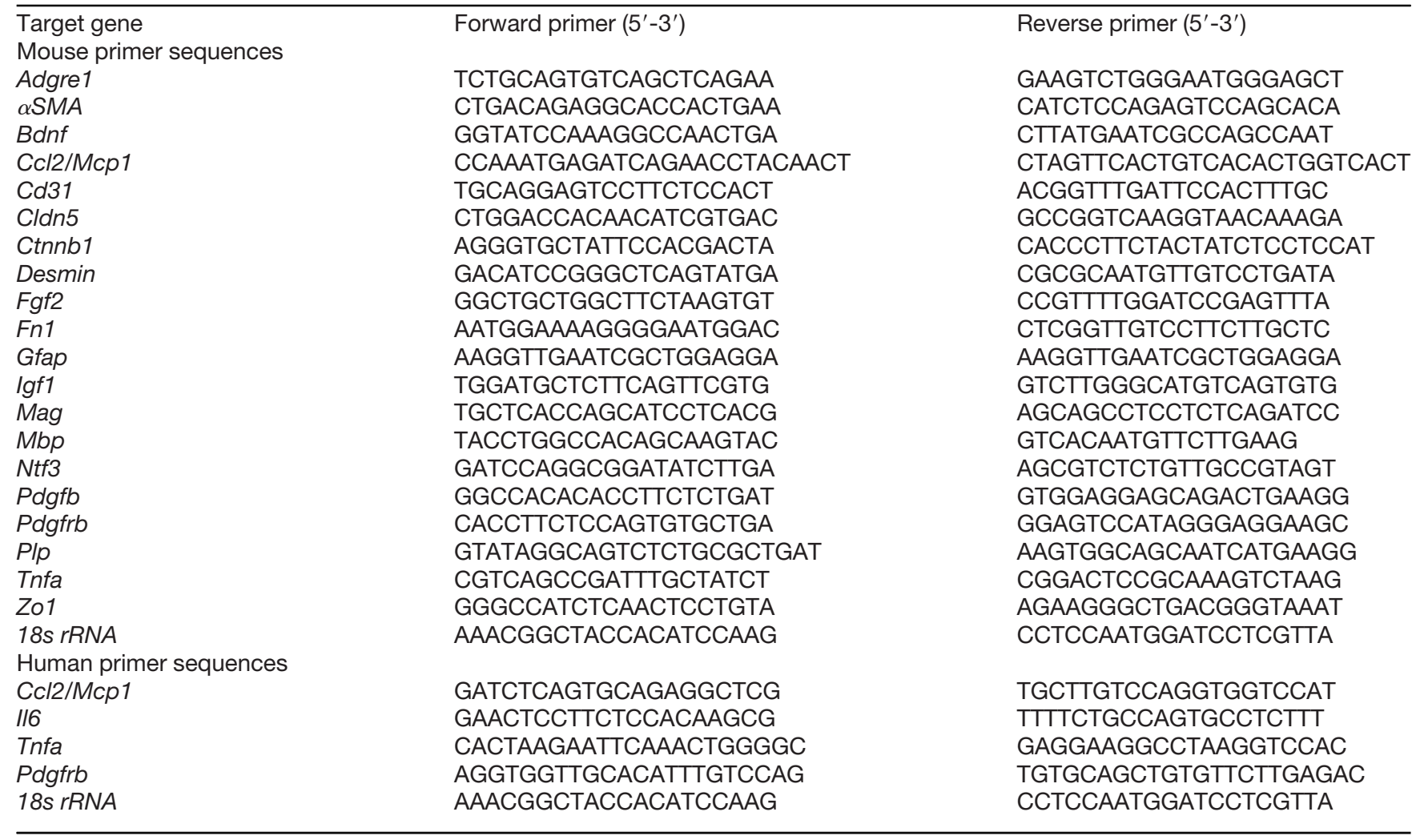

blocked in 5\% skimmed milk for 30 min at room temperature, then incubated at $4^{\circ} \mathrm{C}$ overnight in primary antibodies: anti- $\alpha$ smooth muscle actin (SMA; 1:100; Abcam \#ab7817), anti-CD31 (1:200; BD Biosciences \#550274), and anti-F4/80 (1:100; Abcam \#ab6640). After washing with PBS/Triton X-100, whole mounts were incubated with appropriate secondary antibodies conjugated to Alexa Fluor dyes (Thermo Fisher Scientific) for $30 \mathrm{~min}$. Whole mounts were then washed and embedded in Vectashield DAPI mounting medium (Vector Laboratories \#H1500) in a 35-mm glass dish, cover slipped, then imaged on an inverted confocal microscope (Nikon A1R).

\section{Quantitative polymerase chain reaction (PCR)}

Total RNA was prepared from whole ischemic hemisphere, ischemic area, or cultured cells using TRIzol (Thermo Fisher Scientific \#15596018). Reverse-transcription PCR (RT-PCR) and quantitative real-time PCR were performed as described previously (Makihara et al., 2015). Total RNA was reverse transcribed with a ReverTra Ace qPCR RT kit (Toyobo \#FSQ-101). Using the reverse transcription product as a template, quantitative PCR was performed using a LightCycler (Roche). The mRNA copy numbers were standardized using 18s ribosomal RNA (rRNA) as an internal control. Primer sequences are presented in Table 1.

\section{Immunoblot analysis}

Immunoblot analysis using brain tissues was performed as described previously (Arimura et al., 2012). Membranes were incubated for $1 \mathrm{~h}$ with ECL Advance blocking reagent (GE Healthcare \#RPN418) at room temperature, and probed overnight at $4^{\circ} \mathrm{C}$ with primary antibodies: antiPDGFR $\beta$ (1:1000; CST \#3169), anti- $\beta$-actin (1:3000; Sigma-Aldrich \#A5441), anti-p-AKT (1:1000; CST \#4060), anti-pan-AKT (1:1000; CST \#4691), anti-p-ERK (1:1000; CST \#4370), anti-ERK (1:1000; CST \#4695), anti-p-STAT3 (1:1000; CST \#9145), anti-STAT3 (1:1000; CST \#4904), anti-GFAP (1:1000; CST \#3670), and anti-NeuN (1:1000; Millipore \#MAB377). Membranes were then washed and incubated with secondary antibodies (1:100,000; CST \#7074 or \#7076) for $45 \mathrm{~min}$ at room temperature.

\section{In vivo proliferation and differentiation assay}

EdU (5-ethynyl-2'-deoxyuridine; Thermo Fisher Scientific \#A10044) was used to label proliferating cells. Intraperitoneal EdU injections (50 mg/kg) were performed once daily for $7 \mathrm{~d}$ beginning on day 1 after pMCAO. Mice were killed on day 28 and perfused with $20-\mathrm{ml}$ ice-cold saline followed by $20-\mathrm{ml} 4 \%$ PFA. Brains were removed, and $2-\mathrm{mm}$ coronal slices were postfixed for $1 \mathrm{~h}$ in $4 \%$ PFA, then cryoprotected in $30 \%$ sucrose overnight. Frozen coronal slices were embedded in optimum cutting temperature compound and sectioned at $4 \mu \mathrm{m}$. EdU staining was conducted using a Click-iTTM EdU imaging kit (Thermo Fisher Scientific \#A10338). Briefly, sections were washed three times with PBS. After blocking with a solution of $3 \%$ bovine serum albumin (BSA) in PBS for $30 \mathrm{~min}$ at room temperature, sections were incubated with a reaction cocktail containing Click-iTTM reaction buffer, $\mathrm{CuSO}_{4}$, 
Alexa Fluor 594 Azide and reaction buffer additive for 30 min while protected from light. The sections were washed once with PBS and then stained with antibodies antiGST $\pi$ (1:100; MBL \#312) and anti-NeuN (1:100; Millipore \#MAB377) at $4^{\circ} \mathrm{C}$ overnight. Sections were washed in PBS/Triton X-100, incubated with the secondary antibody for 30 min, washed in PBS/Triton X-100, and then coverslipped with Vectashield DAPI mounting medium (Vector Laboratories). To assess the number of newly generated mature OLs (GST $\pi+/$ EdU + cells) and mature neurons in the penumbra (NeuN+/EdU+ cells) on day 28, three serial sections per animal spaced $400 \mu \mathrm{m}$ apart (from bregma $+1.0 \mathrm{~mm}$ to bregma $-0.2 \mathrm{~mm}$ ) were used. Eight animals per group were analyzed.

\section{Behavioral tests}

Neurological tests were performed pre-pMCAO and 1, $3,7,14,21$, and $28 \mathrm{~d}$ after pMCAO. Sensorimotor deficits were assessed by the rotarod, modified neurological severity score (mNSS), and cylinder tests. The rotarod test was performed as described previously (Gertz et al., 2012). Briefly, mice were placed on a rotating drum accelerating from 4 to $40 \mathrm{rpm}$ over $300 \mathrm{~s}$. The latency to fall off the drum was recorded. Preoperative training was performed for $3 \mathrm{~d}$ with three trials per day, the last three trials serving as preoperative baseline. Postoperative tests were performed three times with an interval of $10 \mathrm{~min}$ between attempts. The mNSS was performed as described previously (Yukami et al., 2015). Neurological function was graded on a scale of 0 to 14 (normal score, 0; maximal deficit score, 14). The mNSS is a composite of motor, sensory, reflex, and balance tests. In the scores for severity of injury, 1 point is awarded for the inability to perform the test or for the lack of a tested reflex; thus, the higher the score, the more severe the injury. The cylinder test was performed as described previously (Tachibana et al., 2017). A mouse was placed in a clear acrylic cylinder $9 \mathrm{~cm}$ in diameter and $20 \mathrm{~cm}$ in height and videotaped during the test. Two mirrors were placed behind the cylinder to record forelimb movements when the mouse was turned away from the camera. Forelimb use during the first contact against the wall after rearing was recorded using previously described criteria in a blinded manner. However, if the right forelimb slipped following the simultaneous use of both forelimbs, a "both" and a "left" movement were recorded. Twenty movements were recorded. The final score $=$ (left forelimb movement - right forelimb movement)/(left forelimb movement + right forelimb movement + both movements). In this test, normal animals would score, on average, 0 . Eight animals per group were analyzed.

\section{Cell culture}

Human brain vascular smooth muscle cells (SMCs) were purchased from ScienCell Research and Laboratories and cultured in SMC medium containing $2 \%$ fetal bovine serum (FBS) and SMC growth supplement. Human brain microvascular pericytes were purchased from ScienCell and cultured in pericyte medium containing $2 \%$ FBS and pericyte growth supplement. Poly-I-lysine (PLL)-coated dishes were prepared (IWAKI). SU16f (10 nM, R\&D Systems \#3304) was used as a selective PDGFR $\beta$ inhibitor. We obtained primary astrocytes and OPCs from ICR mice at postnatal day 1 (P1) using a previously described protocol (Matsuda et al., 2019) with some modifications. To obtain mixed glial cell cultures, cerebral cortices were carefully dissected after stripping away the meninges. The tissue was digested with papain (Sigma-Aldrich \#3125) and triturated with $60 \mathrm{mg} / \mathrm{ml}$ DNase I (Wako \#04726771). After centrifugation (200 g, $5 \mathrm{~min}$ ), the cell pellet was resuspended in $\alpha$-minimum essential medium (MEM), and the suspension was passed through a $40-\mu \mathrm{m}$ cell strainer (BD Falcon \#352340). After centrifugation (200 g, $5 \mathrm{~min}$ ), the cell pellet was resuspended in DMEM containing $10 \%$ FBS. These mixed glial cells were plated in T75 tissue culture flasks. The medium was renewed every 2-3 d. Seven days after plating, microglia and OPCs were detached from astrocyte monolayer sheets by shaking at $200 \mathrm{rpm}$ at $37^{\circ} \mathrm{C}$ for $15-18 \mathrm{~h}$. Detached cells were collected and plated onto uncoated $100-\mathrm{mm}$ culture dishes to eliminate contaminating astrocytes and microglia. After incubation for $30 \mathrm{~min}$, the nonadherent OPCs were collected and plated onto PLL-coated dishes, then cultured with DMEM/F12 (Thermo Fisher Scientific \#12400024) supplemented with insulin (25 mg/ $\mathrm{ml}$, Sigma-Aldrich \#097-06474), apo-transferrin (100 mg/ $\mathrm{ml}$, Sigma-Aldrich \#34401-55), progesterone (20 nM, Sigma-Aldrich \#P0130), putrescine (60 mM, SigmaAldrich \#P5780), sodium selenite (30 nM, Sigma-Aldrich \#S5261), human PDGF-AA (10 ng/ml, Peprotech \#10013A), bFGF (20 ng/ml, Peprotech \#100-18B), and EGF (20 ng/ml, Peprotech \#100-15). Four to $5 \mathrm{~d}$ after plating, the OPCs were used for experiments. After the shake-off procedure for the isolation of microglia and OPCs, the cells were treated with AraC $(5 \mathrm{mM}$, Sigma-Aldrich \#C1768,) for $2 \mathrm{~d}$ to eliminate proliferating cells. Cultures were shaken once more, and then trypsin EDTA solution was added to the flask to obtain the remaining astrocytes, which were transferred to uncoated tissue culture dishes and maintained in DMEM containing 10\% FBS. Two to $3 \mathrm{~d}$ after plating, the astrocytes were used for assays.

\section{CM preparation}

To prepare $\mathrm{CM}$ for treating astrocytes, pericytes were incubated in pericyte medium without FBS and pericyte growth supplement for $48 \mathrm{~h}$ but containing PBS or PDGF-BB (10 nM, Wako \#16424031). Then, CM was collected [referred to as pericyte-CM (PCM)/PBS and PCM/PDGF-BB, respectively]. Primary astrocytes were treated with $\mathrm{PCM}$ and DMEM without serum at a ratio of 1:1 for $24 \mathrm{~h}$. Control medium was prepared with pericyte medium without cultured pericytes and DMEM at a ratio of $1: 1$

\section{In vitro cell proliferation assay}

We performed a cell proliferation assay using the CellTiter96 Aqueous One Solution Cell Proliferation Assay 
Table 2. Physiological data

\begin{tabular}{|c|c|c|c|c|c|c|}
\hline & & Base & 1 & 3 & 7 & 14 \\
\hline Wild type & SBP & $115 \pm 7$ & $98 \pm 7$ & $122 \pm 10$ & $122 \pm 9$ & $117 \pm 9$ \\
\hline Wild type & DBP & $62 \pm 10$ & $56 \pm 11$ & $67 \pm 9$ & $67 \pm 7$ & $65 \pm 9$ \\
\hline Wild type & $\mathrm{HR}$ & $551 \pm 71$ & $527 \pm 106$ & $558 \pm 89$ & $616 \pm 68$ & $616 \pm 83$ \\
\hline$P_{d g f r b}+1-$ & bpm & $572 \pm 64$ & $488 \pm 76$ & $556 \pm 46$ & $572 \pm 55$ & $643 \pm 51$ \\
\hline
\end{tabular}

kit (Promega Corporation \#G3580), containing the tetrazolium dye, MTS, according to the manufacturer's instructions. Briefly, primary astrocytes were seeded onto 96-well plates at $1 \times 10^{4}$ cells/well. The cells were incubated with DMEM containing 10\% FBS for $24 \mathrm{~h}$, then the medium was replaced with CM either with or without anti-IL6 antibody (R\&D Systems \#AF206; $n=6$ per group). In the proliferation assay, $20 \mu$ of the assay reagent was added to each well containing the cells in $100 \mu$ of culture medium. The plate was then incubated for $2 \mathrm{~h}$ in a humidified, $5 \% \mathrm{CO}_{2}$, atmosphere, and the absorbance was recorded at $490 \mathrm{~nm}$ using a microplate reader. Background absorbance was corrected by subtracting the absorbance of the reference at a wavelength of $650 \mathrm{~nm}$. We also examined proliferation of cultured astrocytes by immunofluorescence with EdU staining. EdU was added to culture medium $1 \mathrm{~h}$ before fixation with 4\% PFA in PBS. EdU- and GFAP-double-positive cells were counted in six ROls $(200 \times 200 \mu \mathrm{m})$ in each dish ( $n=6$ dishes).

\section{Scratch assay}

Confluent astrocyte monolayers were scratched once across the diameter of the glass coverslip using a sterile 200- $\mu$ l pipette tip. We removed the culture medium immediately after wounding and replaced it with CM. Quantification of wound area and its closure after $72 \mathrm{~h}$ were analyzed using ImageJ software.

\section{In vitro OPC differentiation assay}

To prepare CM for evaluating OPC differentiation, astrocytes were incubated in DMEM and control PCM, DMEM and PCM/PBS, or DMEM and PCM/PDGF-BB for $24 \mathrm{~h}$. The $\mathrm{CM}$ [referred to as astrocyte-CM (ACM), A-PCM/PBS, or A-PCM/PDGF-BB, respectively] were then collected and centrifuged at $200 \mathrm{~g}$ for $5 \mathrm{~min}$ to remove cells and debris. Primary OPCs were treated with each CM and OPC differentiation medium (N2 medium with $50 \mathrm{ng} / \mathrm{ml} \mathrm{T3}$ and T4, Sigma-Aldrich \#T2877 and \#T2376) at a ratio of 1:2 for 5 or $7 \mathrm{~d}$. Control CM was prepared from an empty dish and diluted with N2 medium in the same way. Medium was replaced every $2 \mathrm{~d}$.

\section{Immunocytochemistry}

Cells were fixed in 4\% PFA and processed for immunostaining. Cells were blocked for $1 \mathrm{~h}$ at room temperature with blocking solution (5\% FBS and $0.3 \%$ Triton X-100). To analyze marker expression profiles the following primary antibodies were used: anti-OLIG2 (1:200; R\&D Systems \#AF2418) and anti-MBP (1:500; Millipore \#MAB386). Stained cells were visualized with a fluorescence microscope (BIOREVO BZ9000). Cell counting was conducted in a blinded manner by randomly selecting six fields for each dish.

\section{Statistical analysis}

Statistical analyses were performed with Student's $t$ test (for two-group comparisons) and one-way ANOVA, followed by a post hoc Bonferroni's comparison test (for comparison of multiple groups) using Prism 8 (GraphPad Software). All data are presented as the mean \pm SEM; $p<$ 0.05 was considered significant. The sample sizes were similar to those reported in previous publications (Tachibana et al., 2017; Komori et al., 2019) and are indicated in the relevant figure legends. No statistical method was used to predetermine sample size.

\section{Results}

Reduction in the size of MAP2-negative infarct areas in the subacute phase is attenuated in Pdgfrb $^{+/-}$mice

We subjected wild-type and Pdgfrb $^{+/-}$mice to pMCAO. Immunoblot analysis demonstrated that the baseline expression level of PDGFR $\beta$ was lower in brain homogenates of Pdgfrb ${ }^{+/-}$mice by $\sim 80 \%$ ( $p<0.001$, unpaired $t$ test; Extended Data Fig. 1-1). We confirmed by immunoblot analysis that PDGFR $\beta$ was abundant in cultured pericytes, but not in cultured astrocytes or cortical neurons (Extended Data Fig. 1-2). We also confirmed that systolic BP (SBP), diastolic BP (DBP) and HR were not different between wild-type and Pdgfrb ${ }^{+/}$mice $(n=10$, each group; Table 2). However, post-stroke body-weight loss was prolonged in Pdgfrb ${ }^{+/-}$mice, compared with wildtype mice (day $1, p=0.317$; day $3, p=0.559$; day $7, p=$ 0.055 ; day $14, p=0.006$; day $21, p<0.001$; day $28, p<$ 0.001 , unpaired $t$ test; Extended Data Fig. 1-3).

Immunohistochemically, MAP2-negative areas, including infarct and edema areas, were comparable between wild-type and Pdgfrb ${ }^{+/-}$mice on day 1 after $\operatorname{pMCAO}(p=$ 0.741 , unpaired $t$ test); however, they were significantly larger in $\mathrm{Pdgfrb}^{+/-}$mice compared with wild-type mice from day 7 to 28 (day $7, p=0.019$; day $14, p=0.015$; day $28, p=0.001$, unpaired $t$ test; Fig. $1 A, B$ ). Similarly, infarct volumes were comparable between Pdgfrb $^{+/-}$and wildtype mice on day 1; however, they were significantly larger in Pdgfrb $^{+/}$mice on day 28 ( $p=0.033$, ANOVA; Fig. 1C). These data suggested that brain edema was greater in Pdgfrb ${ }^{+/-}$mice on days 7 and 14. Furthermore, tissue atrophy was significantly larger in Pdgfrb ${ }^{+/-}$mice on day 28 ( $p=0.023$, unpaired $t$ test; Fig. 1D). However, 
A

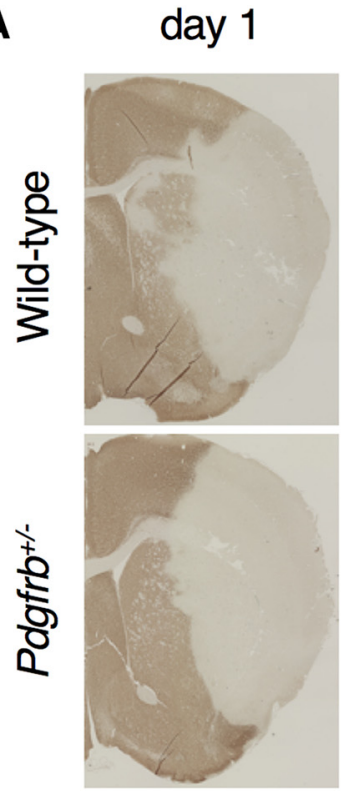

B

MAP2-negative areas

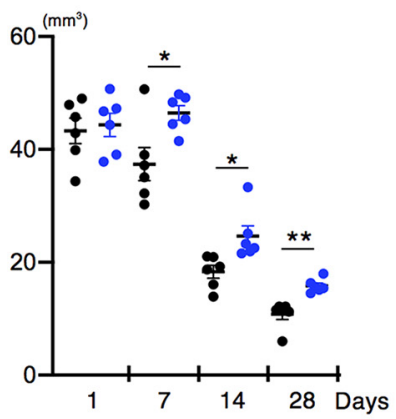

day 7

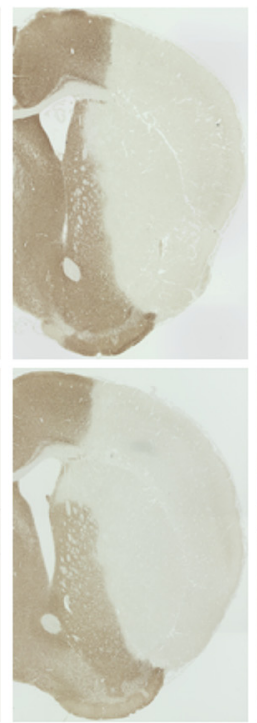

C

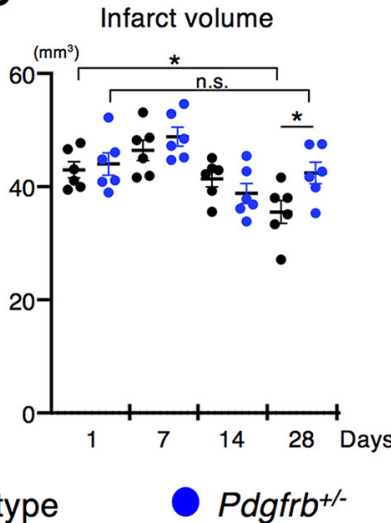

day 14
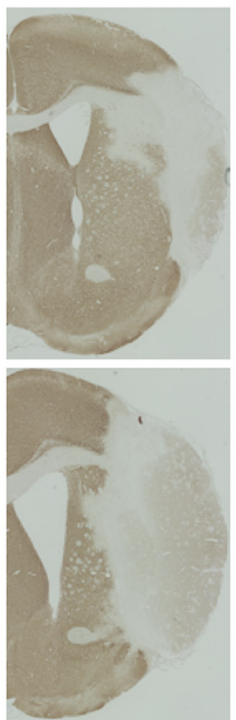

D day 28
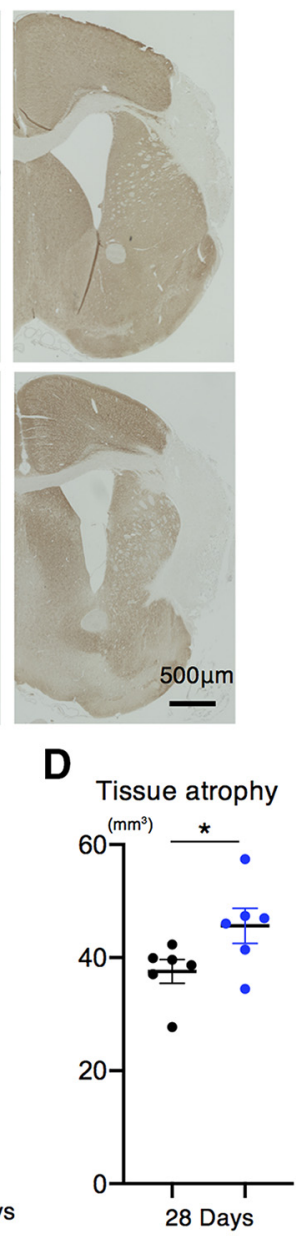

Figure 1. Reduction of MAP2-negative areas and infarct volume in the subacute phase is attenuated in $P d g f r b^{+/-}$mice. $\boldsymbol{A}_{\text {, MAP2 }}$ staining on days $1,7,14$, and 28 after pMCAO in wild-type and Pdgfrb ${ }^{+/-}$mice (scale bar, $500 \mu \mathrm{m}$ ). B, Quantification of MAP2-negative areas on days $1,7,14$, and 28 after pMCAO ( $n=6$, each group). $\boldsymbol{C}$, Quantification of infarct volume on days $1,7,14$, and 28 after pMCAO ( $n=6$, each group). $\boldsymbol{D}$, Quantification of tissue atrophy on day 28 after pMCAO ( $n=6$, each group). Data are shown as the mean \pm SEM. $\boldsymbol{B}, \boldsymbol{D}, * p<0.05$ and $* * p<0.01$, unpaired $t$ test. $\boldsymbol{C}, * p<0.05$, one-way ANOVA followed by Bonferroni's post hoc test. n.s.: not significant.

we should note that the difference of these parameters between the groups was small.

\section{Leptomeningeal arteriogenesis accompanied by monocyte/macrophage accumulation that leads to recovery of blood flow within ischemic areas is attenuated in $\mathrm{Pdgfrb}^{+/-}$mice}

We next examined post-stroke recovery of CBF within ischemic areas. Although CBF in ischemic areas, as assessed by high-resolution 2D laser speckle flowmetry, was reduced equally in wild-type and $\mathrm{Pdgfrb}^{+/-}$mice $(47 \pm 7 \%$ vs $46 \pm 6 \%, p=0.942$, unpaired $t$ test) on day 1 after pMCAO, its recovery was significantly suppressed in Pdgfrb $^{+/-}$mice compared with wild-type mice on day 7 (70 $\pm 11 \%$ vs $82 \pm 4 \%, p=0.005$, unpaired $t$ test), day 14 (75 $\pm 8 \%$ vs $93 \pm 5 \%, p<0.001$, unpaired $t$ test), and day $28(80 \pm 9 \%$ vs $94 \pm 3 \%, p<0.001$, unpaired $t$ test; $n=12$; Fig. $2 A, B)$.

We then examined the extent of leptomeningeal arteriogenesis after PMCAO. The number and the average diameter of leptomeningeal anastomoses between the distal MCA and the distal anterior cerebral artery (ACA) at baseline, as assessed by a latex perfusion method, were similar between wild-type (11 \pm 2 vessels, $20.8 \pm 4.1 \mu \mathrm{m}$; Fig. $2 \mathrm{Ca}, e)$ and $P$ dgfrb ${ }^{+/-}$mice $(10 \pm 1$ vessels, $20.8 \pm 3.7$ $\mu \mathrm{m}$; Fig. 2Cc,g; $p=0.872, p=0.931, n=7$, ANOVA; Fig. $2 D, E$ ). On day 7 after pMCAO, the MCA territory was retrogradely perfused through enlarged leptomeningeal collaterals (Fig. $2 \mathrm{Cb}, d)$. The average diameter of collaterals was significantly greater in wild-type $(46.6 \pm 11.0 \mu \mathrm{m}$; Fig. 2Cf) compared with Pdgfrb ${ }^{+/-}$mice $(35.4 \pm 8.2 \mu \mathrm{m}$; Fig. $2 C h ; n=8, p=0.008$, ANOVA; Fig. 2D), while the number of collaterals was not significantly different 
A

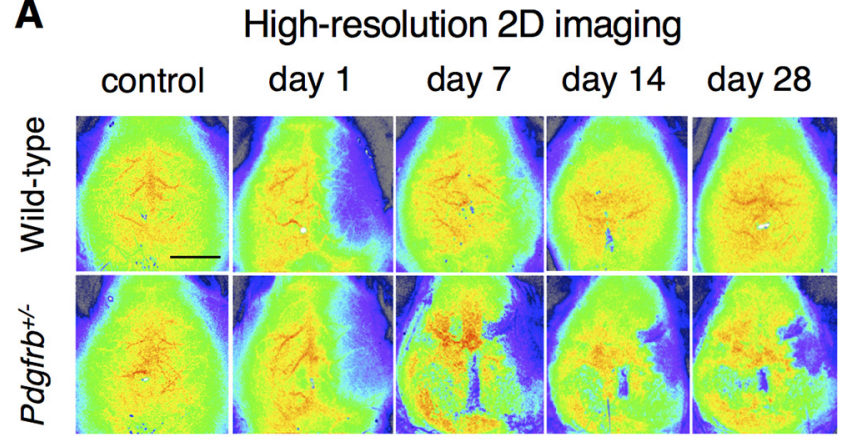

B

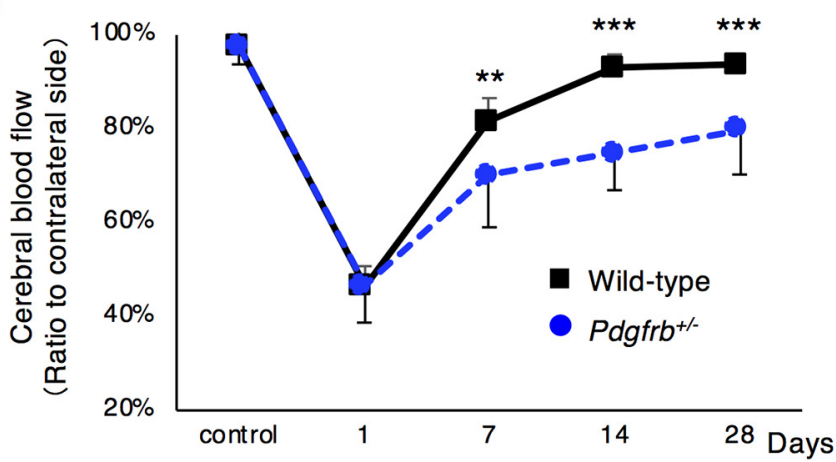

C

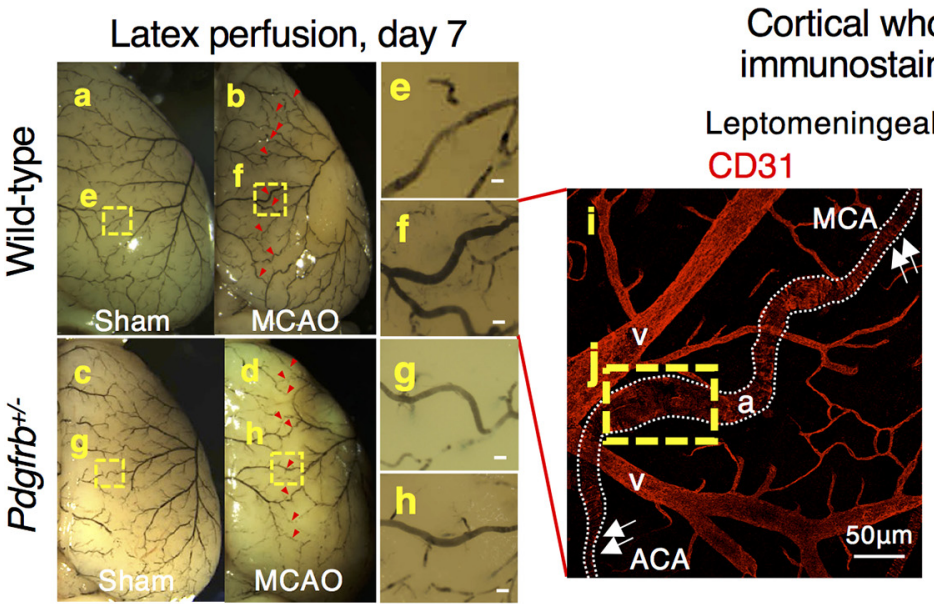

Cortical whole mount immunostaining, day 7

Leptomeningeal anastomoses

MCA

$\mathbf{F}$ ischemic hemisphere, day 7

Wild-type - Pdgrrb+-

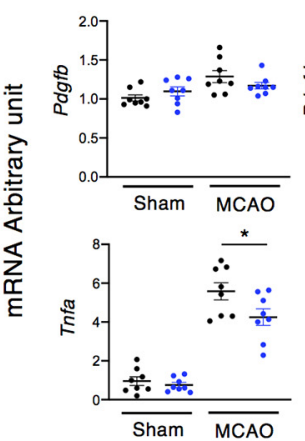

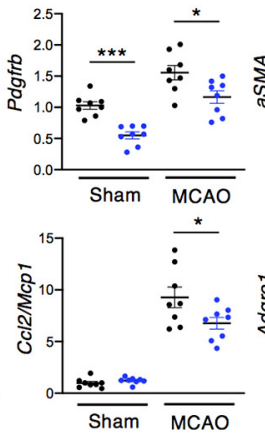
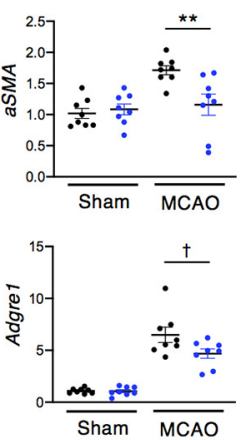

aSMA CD31
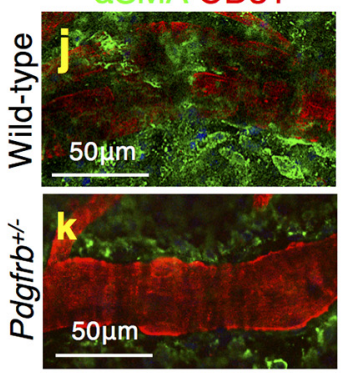

G Cortical whole mount immunostaining, day 7
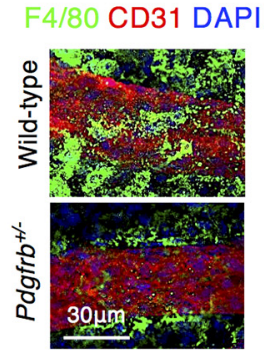

D

Figure 2. Leptomeningeal arteriogenesis and recovery of CBF in ischemic areas after pMCAO are attenuated in Pdgfrb ${ }^{+/-}$mice. $\boldsymbol{A}$, Images of CBF just under the cortical surface, as assessed by laser speckle flowmetry, in control and on days 1, 7, 14, and 28 after pMCAO in wild-type and $\mathrm{Pdgfrb}^{+/-}$mice (scale bar, $2 \mathrm{~mm}$ ). $\boldsymbol{B}$, Temporal profiles of CBF quantification on days 1, 7, 14, and 28 after pMCAO ( $n=12$, each group). C Macroscopic images of leptomeningeal anastomoses, assessed by a latex perfusion method, at baseline $(\boldsymbol{a}, \boldsymbol{c})$ and on day $7(\boldsymbol{b}, \boldsymbol{d})$ after pMCAO in wild-type and Pdgfrb ${ }^{+/-}$mice (scale bar, $\left.50 \mu \mathrm{m}\right)$. Arrowheads indicate the leptomeningeal anastomoses vessels $(\boldsymbol{b}, \boldsymbol{d})$. Magnified images of the dotted squares $(\boldsymbol{e}-\boldsymbol{h})$ in $\boldsymbol{a}-\boldsymbol{d}$ are shown in the right panels. Cortical whole mount immunostaining of leptomeningeal anastomoses with CD31 (red) on day 7 after pMCAO is shown in $\boldsymbol{i}$ (a: artery, v: vein). Arrows indicate the pre-existing ACA and MCA. The magnified image of the yellow dotted square (j) is shown in the right panel. Double immunofluorescence labeling with CD31 (red) and $\alpha$ SMA (green) in anastomosed vessels on day 7 after pMCAO in wild-type (j) and $\mathrm{Pdgfrb}^{+/-}$mice $(\boldsymbol{k})$ is shown (scale bar, $\left.50 \mu \mathrm{m}\right)$. $\boldsymbol{D}$, Diameter of anastomotic vessels at baseline and on day 7 after pMCAO (baseline, $n=7$ each group; pMCAO, $n=8$ each group). $\boldsymbol{E}$, Number of anastomotic vessels at baseline and on day 7 after pMCAO (baseline, $n=7$ each group; pMCAO, $n=8$ each group). $\boldsymbol{F}$, mRNA levels of arteriogenesis-related genes in non-infarct hemisphere and ischemic hemisphere on day 7 ( $n=8$, each group). G, Cortical whole mount immunostaining with CD31 (red), F4/80 (green), and DAPI (blue) in wild-type and $\mathrm{Pdgfrb}^{+/-}$mice (scale bar, $50 \mu \mathrm{m}$ ). $\boldsymbol{H}$, Quantitative PCR for Ccl2/Mcp1 and Tnfa in cultured VSMCs at baseline (black), with PDGF-BB (10 ng/ml; red), and with SU16f-pretreated PDGF-BB (100 nmol//; blue; $n=6$, each 
continued

group). Data are shown as the mean \pm SEM. $\boldsymbol{B}, \boldsymbol{F},{ }^{\dagger} p<0.1, * p<0.05, * * p<0.01$, and $* * * p<0.001$, unpaired $t$ test. $\boldsymbol{D}-\boldsymbol{E}, \boldsymbol{H}$, ${ }^{\dagger} p<0.1$, $* p<0.05$, $* * p<0.01$, and $* * * p<0.001$, one-way ANOVA followed by Bonferroni's post hoc test.

between the groups $(10 \pm 1$ vessels vs $10 \pm 1$ vessels, $n=8, p=0.376$, ANOVA; Fig. 2E). Cortical whole mount staining with CD31 confirmed that the diameter of anastomosed points was increased compared with the surrounding pre-existing ACA and MCA (arrows) on day 7 (Fig. 2Ci). Double staining with CD31 and $\alpha$ SMA showed that greater numbers of $\alpha \mathrm{SMA}$-positive SMCs were accumulated at the anastomosed points in wild-type mice compared with $\mathrm{Pdgfrb}^{+/-}$mice (Fig. 2Cj,k).

We then investigated mRNA expression of arteriogenesis-related molecules in the ischemic hemisphere. Poststroke expression of aSMA and Pdgfrb was significantly lower (aSMA, $p=0.009$; Pdgfrb, $p=0.022$, unpaired $t$ test), while that of platelet-derived growth factor subunit B (Pdgfb) was not altered ( $p=0.212$, unpaired $t$ test), on day 7 in Pdgfrb ${ }^{+/-}$mice (Fig. 2F). Furthermore, mRNA levels of Tnfa and Ccl2/Mcp1, a representative pro-inflammatory cytokine and chemokine that recruit monocytes/ macrophages, were significantly lower on day 7 in Pdgfrb $^{+/-}$mice (Tnfa, $p=0.049 ;$ Ccl2/Mcp1, $p=0.048$, unpaired $t$ test; Fig. 2F). Consistently, immunofluorescence staining demonstrated that the recruitment of F4/80-positive monocytes/macrophages around anastomosed points was attenuated in $\mathrm{Pdgfrb}^{+/-}$mice, compared with wild-type mice (Fig. 2G).

We further demonstrated using cultured vascular SMCs that treatment with PDGFB homodimer (PDGF-BB) significantly increased the mRNA levels of Ccl2/Mcp1 $(p<$ 0.001 , ANOVA) and Tnfa ( $p=0.009$, ANOVA), while pretreatment of the cells with SU16f, an inhibitor of PDGFR $\beta$, abolished the effects of PDGF-BB (Cc/2/Mcp1: $p=0.029$; Tnfa: $p=0.092$, ANOVA; Fig. $2 H$ ). These findings indicated that SMCs, same lineage cells to pericytes, expressed PDGFR $\beta$ and produced molecules that recruit monocytes/macrophages, which can promote remodeling of collateral vessels leading to arteriogenesis (Scholz et al., 2000; Sugiyama et al., 2011).

\section{Intrainfarct angiogenesis and fibrosis are attenuated in Pdgfrb ${ }^{+/-}$mice}

Consistent with the concept that reperfusion after ischemia promotes the repair process within infarct areas (Tachibana et al., 2017), intrainfarct fibrotic formation, as assessed by immunohistochemical staining with pericyte markers PDGFR $\beta$ and CD13 (Montagne et al., 2018), gradually expanded from day 7 to 28 and led to the shrinkage of infarct areas in wild-type mice (Figs. $1 A, 3 A$, $B)$. However, CD13-positive fibrotic formation was significantly attenuated in Pdgfrb ${ }^{+/-}$mice (control, $p=0.607$; day $7, p=0.337$; day $14, p=0.009$; day $28, p=0.007$, unpaired $t$ test; Fig. $3 B, C)$. Macroscopic observation of the brain surface supported impaired scar formation in ischemic areas in Pdgfrb ${ }^{+/-}$mice on day 28 after pMCAO (Fig. $3 D$ ). The breakdown of the BBB within ischemic areas, as assessed by microscopic extravasation of fibrin $(p=$
0.005, unpaired $t$ test; Fig. 3E,F) and macroscopic extravasation of Evans blue dye (Fig. $3 G$ ), was greater on day 28 in Pdgfrb ${ }^{+/-}$mice. We further demonstrated that the number of CD31-positive endothelial cells within infarct areas on day 28 was significantly smaller in $P$ dgfrb ${ }^{+/}$ mice $(2.4 \pm 0.8 \%$ vs $3.9 \pm 1.4 \%$ in wild type, $p=0.024$, unpaired $t$ test; Fig. $3 H, I)$, indicating that PDGFR $\beta$ also participated in intrainfarct angiogenesis leading to recovery of CBF after pMCAO.

To elucidate the significance of PDGFR $\beta$-positive fibrotic lesions, we examined gene expression in non-infarct control and within ischemic areas on days 7 and 14 in wild-type and $\mathrm{Pdgfrb}^{+/-}$mice. The baseline expression of genes examined here, except Pdgfrb, was not significantly different between wild-type and $\mathrm{Pdgfrb}^{+/-}$mice (Fig. 3J). The expression of genes related to endothelial cells (Cd31), pericytes [Des (Desmin)], growth factors (Pdgfb and Fgf2), tight-junction related proteins [Cldn5 (Claudin 5) and Zo1], and extracellular matrix proteins [Fn1 (fibronectin 1)] was significantly decreased in Pdgfrb $^{+/-}$mice on day 14 after pMCAO (Fig. 3J). Furthermore, the expression of Bdnf and Ntf3, representative neurotrophic factors, was significantly decreased in Pdgfrb $^{+/-}$mice (Fig. 3J), indicating that PDGFR $\beta$-mediated repair in ischemic areas may exert some positive effects on peri-infarct neural reorganization during the subacute phase after pMCAO.

\section{PDGFR $\beta$-mediated repair enhances peri-infarct astrogliosis}

Consistent with previous studies (Shen et al., 2012; Makihara et al., 2015), we found that peri-infarct astrogliosis was significantly attenuated in $P d g f r b^{+/-}$mice $(p=$ 0.018 , unpaired $t$ test; Fig. $4 A$ ) with a clear boundary to PDGFR $\beta$-positive fibrotic areas (Fig. 4B). In contrast, the extent of IBA1-positive microglia/macrophage, localized in peri-infarct area, was not significantly different between wild-type and Pdgfrb ${ }^{+/-}$mice on day 28 after pMCAO $(p=0.465$, unpaired $t$ test; Extended Data Fig. 4-1A,B). Immunofluorescence staining demonstrated that IBA1positive microglia/macrophage did not appear to express PDGFR $\beta$ (Extended Data Fig. 4-1C).

To test a possible interaction between PDGFR $\beta$-positive cells and GFAP-positive reactive astrocytes, we examined the effects of PCM treated with either PBS (PCM/ PBS) or PDGF-BB (PCM/PDGF-BB) on cultured astrocytes (Fig. 4C). While PCM/PBS increased the number of astrocytes, PCM/PDGF-BB further enhanced the growth effect of PCM/PBS (PCM/PBS, $p=0.002$; PCM/PDGF$\mathrm{BB}, p<0.001$, ANOVA; Fig. 4D). A cell proliferation assay using EdU confirmed the growth effects of PCM/PBS and PCM/PDGF-BB on cultured astrocytes (PCM/PBS, $p=0.08$; PCM/PDGF-BB, $p=0.003$; ANOVA; Fig. 4E). Moreover, PCM/PBS enhanced astrocyte growth and migration after culture dishes were scratched, while PCM/ 
A

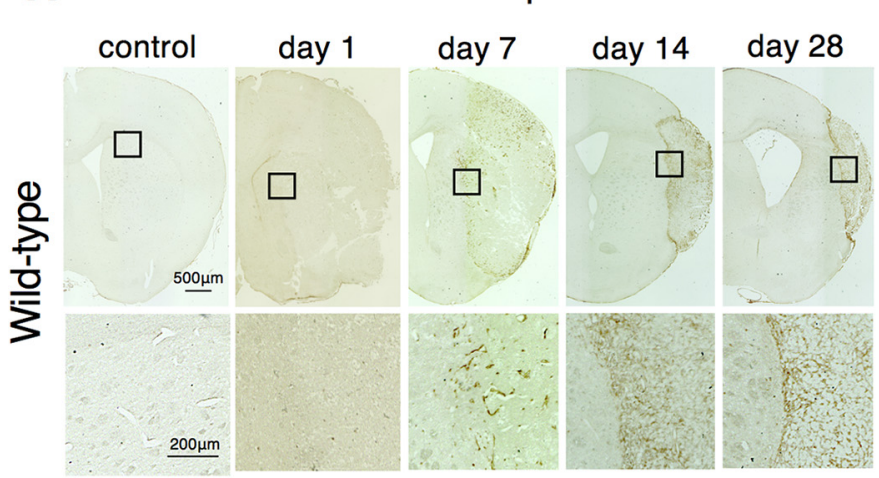

B control day 7 day 14 day 28
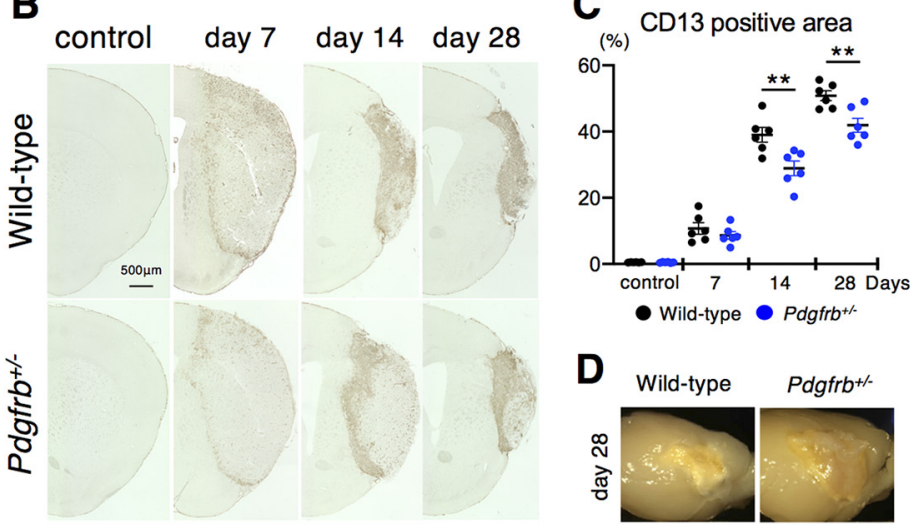

E<smiles></smiles>

infarction

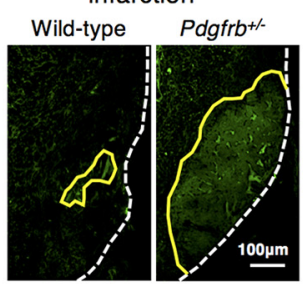

$\mathbf{F}$

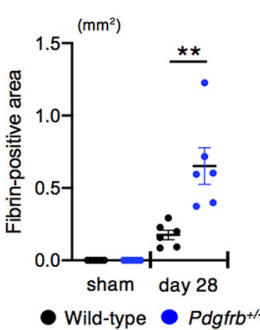

$\mathbf{G}$ day 28 Evans Blue

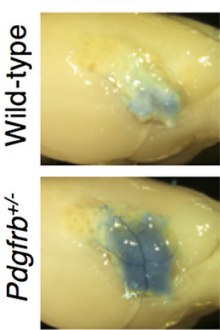

\section{H Wild-type \\ CD31, day 28 \\ Pdgfrb ${ }^{+/-}$}
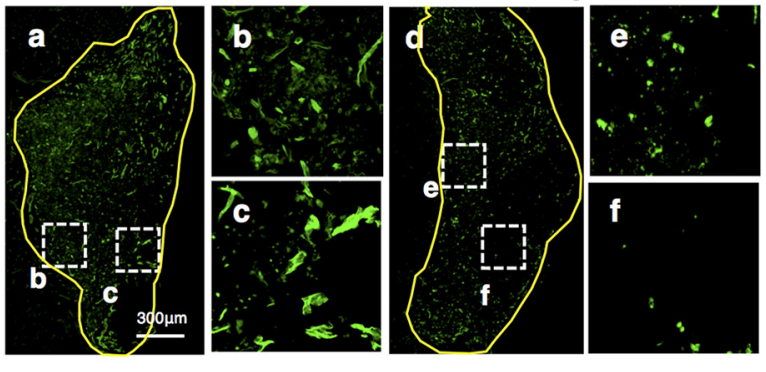

(\%)

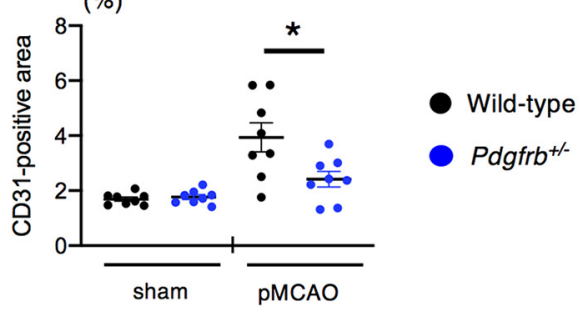

J

ischemic area (mRNA Arbitrary unit)

Wild-type

Pdgfrb $^{+-}$
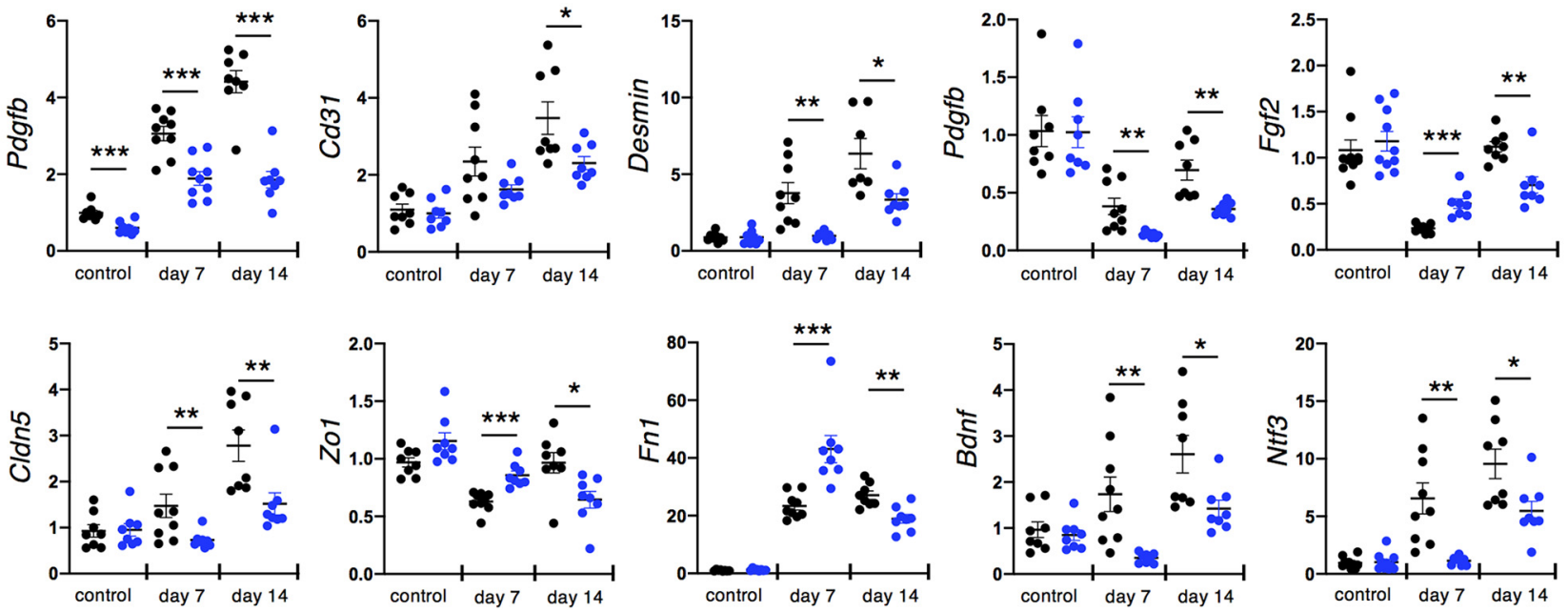

Figure 3. PDGFR $\beta$ is important for intrainfarct angiogenesis and fibrosis. $\boldsymbol{A}$, Immunohistochemistry of PDGFR $\beta$ at baseline and on days $1,7,14$, and 28 after pMCAO (scale bar, $500 \mu \mathrm{m}$ ). Magnified images in the squares are shown at the bottom. $\boldsymbol{B}$, Immunohistochemistry of CD13, a marker of pericyte and pericyte-derived cells, in control and on days 7, 14, and 28 after pMCAO (scale bar, $500 \mu \mathrm{m})$. C, Quantification of CD13-positive areas in control and on days 7, 14, and 28 after pMCAO in wild-type and Pdgfrb $^{+/-}$mice $(n=6$, each group). $\boldsymbol{D}$, Representative macroscopic observation of the brain surface on day 28 after pMCAO $(n=$ 10). $\boldsymbol{E}$, Fibrin staining on day 28 after pMCAO (scale bar, $100 \mu \mathrm{m}$ ). $\boldsymbol{F}$, Quantification of fibrin-positive areas in mice on day 28 after pMCAO and in sham-operated mice ( $n=6$, each group). $\mathbf{G}$, Representative macroscopic images of Evans blue dye leakage in the brain on day 28 after pMCAO $(n=6)$. $\boldsymbol{H}$, Immunofluorescence labeling of CD31 on day 28 after pMCAO in wild-type (a) and Pdgfrb $^{+/-}$mice (d; scale bar, $\left.300 \mu \mathrm{m}\right)$. Magnified images in the dotted squares $(\boldsymbol{b}, \boldsymbol{c}, \boldsymbol{e}, \boldsymbol{f})$ are shown to the right. $\boldsymbol{I}$, Quantification of 
continued

intrainfarct CD31 density in mice on day 28 after pMCAO and in sham-operated mice ( $n=8$, each group). $\boldsymbol{J}$, Quantitative PCR of genes expressed in non-infarct control and within infarct areas on days 7 and 14 after pMCAO in wild-type (black) and Pdgfrb ${ }^{+/-}$ mice (blue; $n=8$, each group). Data are shown as the mean \pm SEM. $\boldsymbol{C}, \boldsymbol{F}, \boldsymbol{I}, * p<0.05$, and $* * p<0.01$, unpaired $t$ test. $\boldsymbol{J}$, $* p<$ $0.05, * * p<0.01$, and $* * * p<0.001$, one-way ANOVA followed by Bonferroni's post hoc test.
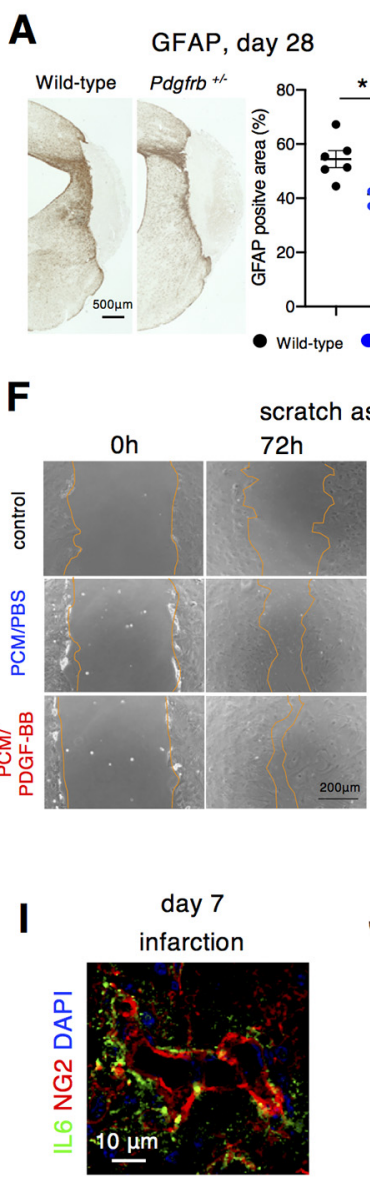

B
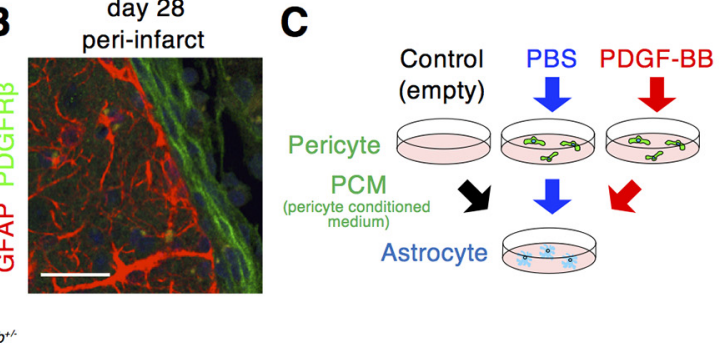

G

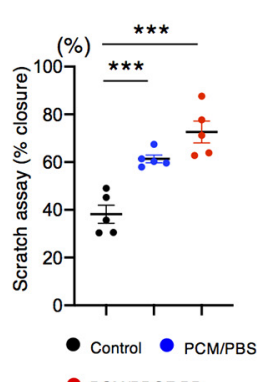

- PCM/PDGF-BB

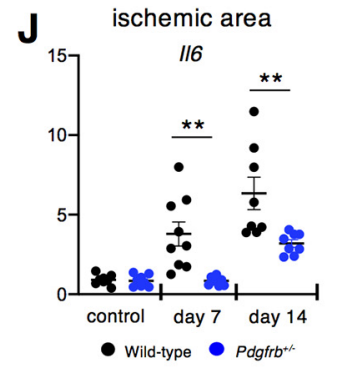

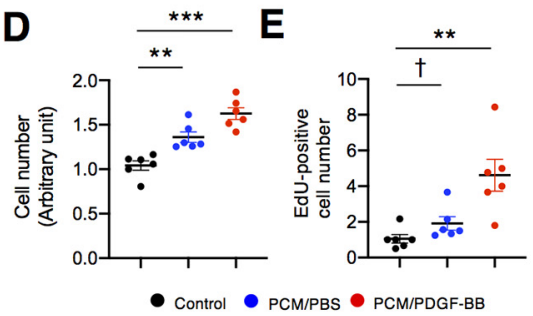
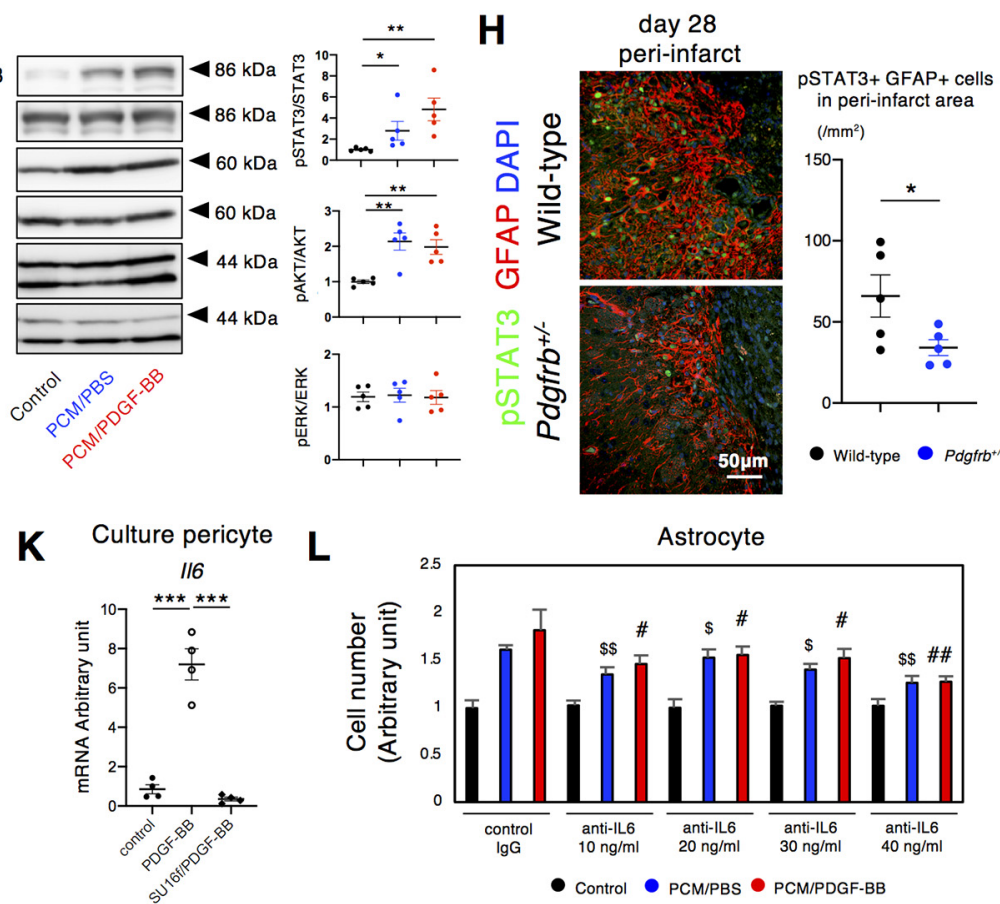

Astrocyte

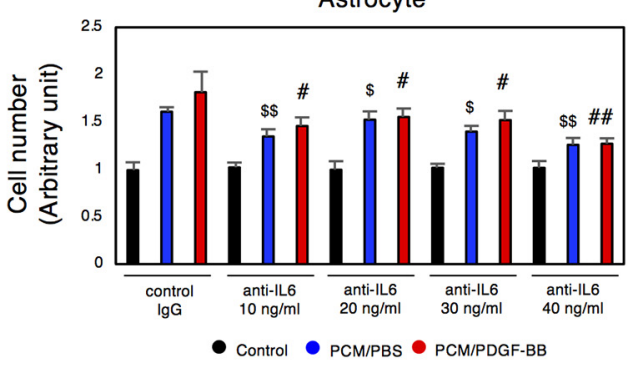

Figure 4. Pericyte-astrocyte interaction in peri-infarct areas after pMCAO. $\boldsymbol{A}$, Immunohistochemistry for GFAP (scale bar, $500 \mu \mathrm{m}$ ) and quantification of GFAP-positive areas on day 28 after pMCAO in wild-type and Pdgfrb ${ }^{+/-}$mice $(n=6$, each group). B, Double immunofluorescence labeling of GFAP (red) and PDGFR $\beta$ (green; scale bar, $20 \mu \mathrm{m}$ ) on day 28 after pMCAO. PDGFR $\beta$-positive fibrotic lesion and GFAP-positive astrogliosis form a clear boundary. $\boldsymbol{C}$, Experimental scheme for phenotypic changes induced in astrocytes by pericyte culture medium (black, control), PCM treated with PBS (blue, PCM/PBS) or PDGF-BB (10 ng/ml; red, PCM/ PDGF-BB). D, MTT assay for astrocytes after treatment with PCM for $24 \mathrm{~h}$ ( $n=6$, each group). $\boldsymbol{E}$, Astrocyte proliferation assay as assessed by immunofluorescence of EdU after treatment with PCM for $24 \mathrm{~h}$ ( $n=6$, each group). $\boldsymbol{F}$, Astrocyte scratch assay after treatment with PCM for $72 \mathrm{~h}$ (scale bar, $200 \mu \mathrm{m}$; left). Quantification of wound recovery ( $n=5$, each group). $\mathbf{G}$, Immunoblot analyses of STAT3 and phospho-STAT3, AKT and phospho-AKT, and ERK and phospho-ERK in astrocytes after treatment with CM for 30 $\min (n=5$, each group). $\boldsymbol{H}$, Representative immunofluorescence labeling for PSTAT3 (green) and GFAP (red), and DAPI (blue) in the peri-infarct area after pMCAO in wild-type and Pdgfrb $^{+/}$mice on day 28 (scale bar, $50 \mu \mathrm{m}$ ). Quantification of the number of pSTAT3 and GFAP double-positive cells are evaluated ( $n=5$, each group). $I$, Double immunofluorescence labeling of NG2 (red) and IL6 (green; scale bar, $10 \mu \mathrm{m}$ ) on day 7 after pMCAO. J, Quantitative PCR of I/6 expression in non-infarct hemisphere and within infarct areas on days 7 and 14 after pMCAO in wild-type (black) and Pdgfrb ${ }^{+/-}$mice (blue; $n=8$, each group). $K$, Expression change of $I / 6$ in pericytes treated with PDGF-BB in the presence or absence of SU16f ( $n=4$, each group). $\boldsymbol{L}$, MTT assay for astrocytes treated with PCM in the presence of anti-IL6 antibody (10-40 ng/ml; $n=6$, each group). Data are shown as the mean \pm SEM. $\boldsymbol{A}, \boldsymbol{H}$, $\boldsymbol{J}, * p<0.05$, and $* * p<0.01$, unpaired $t$ test. $\boldsymbol{D}-\mathbf{G}, \boldsymbol{K}, \boldsymbol{L},{ }^{\dagger} p<0.1, * p<0.05, * * p<0.01$, and $* * * p<0.001 ;{ }^{\dagger} p<0.05$ and ${ }^{\$ \$} p<$ 0.01 versus control IgG with PCM/PBS; and ${ }^{\#} p<0.05$ and ${ }^{\# \#} p<0.01$ versus control IgG with PCM/PDGF-BB, one-way ANOVA followed by Bonferroni's post hoc test. 

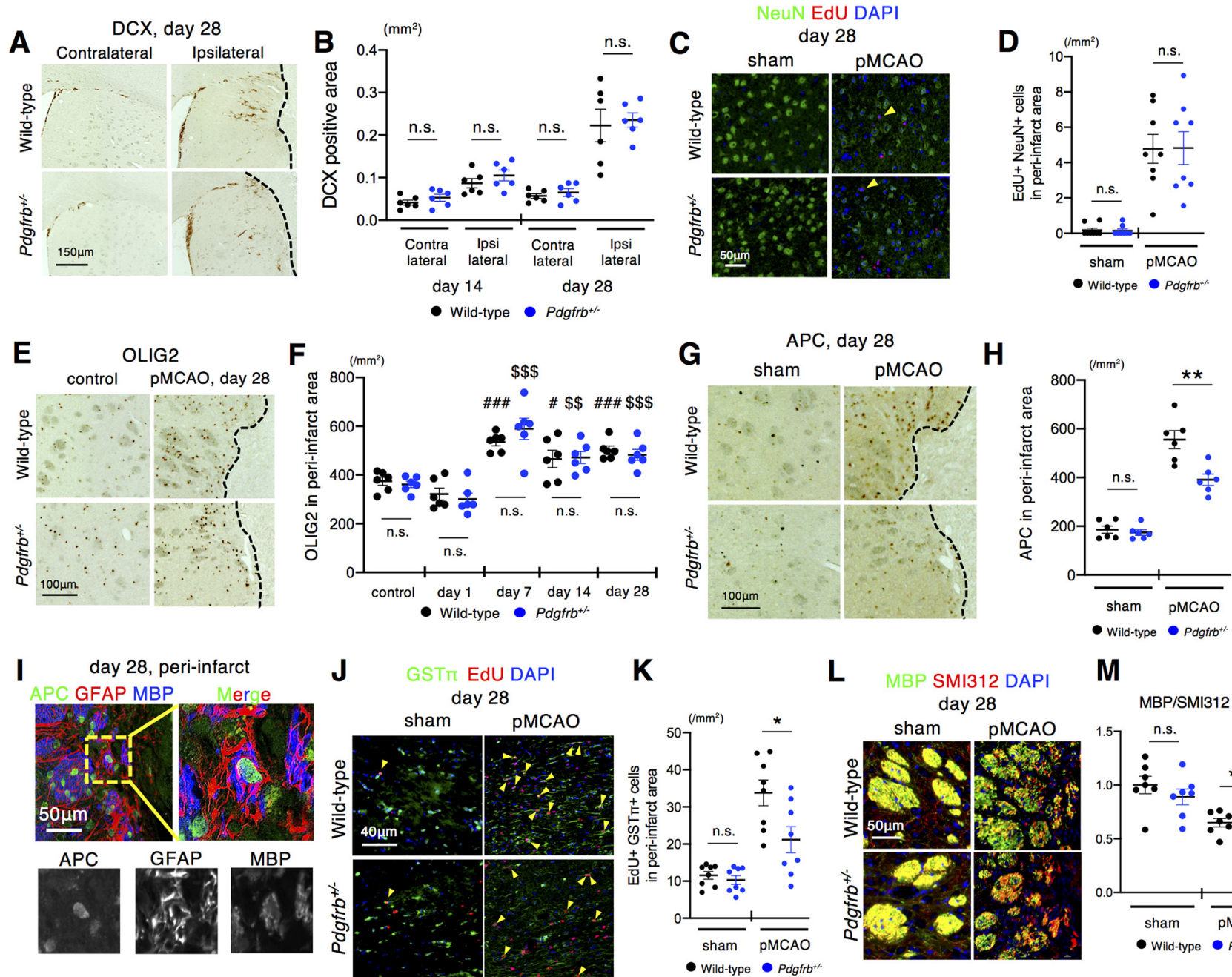

M

MBP/SMI312 ratio

Figure 5. Peri-infarct oligodendrogenesis but not neurogenesis is attenuated in Pdgfrb ${ }^{+/}$mice. $\boldsymbol{A}$, Immunohistochemistry for DCX in the SVZ and striatum on day 28 after pMCAO in wild-type and Pdgfrb ${ }^{+/}$mice (scale bar, $150 \mu \mathrm{m}$ ). B, Quantification of the DCX-positive areas in the ipsilateral and contralateral hemisphere on days 14 and 28 after pMCAO in wild-type (black) and Pdgfrb ${ }^{+/-}$mice (blue; $n=6$, each group). $\boldsymbol{C}$, Double immunofluorescence labeling of NeuN (green) and EdU (red) in the peri-infarct areas on day 28 after pMCAO and in sham-operated mice. Arrowheads indicate NeuN and EdU double-positive newborn mature neurons (scale bar, $50 \mu \mathrm{m})$. $\boldsymbol{D}, \mathrm{Quantification}$ of the number of double-positive cells in peri-infarct areas on day 28 after pMCAO and in sham-operated mice ( $n=8$, each group). $\boldsymbol{E}$, Immunohistochemistry for OLIG2 in sham-operated mice and in peri-infarct areas on day 28 after pMCAO in wild-type and Pdgfrb ${ }^{+/-}$mice (scale bar, $100 \mu \mathrm{m}) . \boldsymbol{F}$, Quantification of the number of OLIG2-positive cells in peri-infarct areas on days 1, 7, 14, and 28 after pMCAO in wild-type (black) and Pdgfrb ${ }^{+/}$mice (blue; $n=6$, each group). G, Immunohistochemistry for APC in sham-operated mice and in peri-infarct areas on day 28 after pMCAO (scale bar, $100 \mu \mathrm{m}$ ). $\boldsymbol{H}$, Quantification of the number of APC-positive cells in peri-infarct areas on day 28 after pMCAO ( $n=6$, each group). I, Triple immunofluorescence labeling with APC (green), GFAP (red) and MBP (blue) in peri-infarct areas (striatum) on day 28 after PMCAO. Magnified images of the dotted square are shown below and in the right panel (scale bar, $50 \mu \mathrm{m}$ ). $\mathbf{J}, \mathrm{Double}$ immunofluorescence labeling of GST $\pi$ (green) and EdU (red) in sham-operated mice and in the peri-infarct areas on day 28 after pMCAO. Arrowheads indicate GST $\pi$ and EdU double-positive newborn mature OLs (scale bar, $40 \mu \mathrm{m}$ ). $\boldsymbol{K}$, Quantification of the number of doublepositive cells in peri-infarct areas on day 28 after pMCAO $(n=8$, each group). $\boldsymbol{L}$, Double immunofluorescence labeling of MBP (green) and the pan-axonal neurofilament marker, SMI312 (red), in sham-operated mice and in peri-infarct striatum on day 28 after pMCAO (scale bar, $50 \mu \mathrm{m}) . \boldsymbol{M}$, Quantification of MBP/SMI312 ratio in the peri-infarct striatum on day 28 after pMCAO ( $n=7$, each group). Data are shown as the mean \pm SEM. $\boldsymbol{B}, \boldsymbol{D}, \boldsymbol{H}, \boldsymbol{K}, \boldsymbol{M}, * p<0.05$ and $* * p<0.01$, unpaired $t$ test. $\boldsymbol{F},{ }^{\#} p<0.05$ and ${ }^{\# \# \# ~} p<0.001$ vs. control wild-type mice, ${ }^{\$ \$} p<0.01$ and ${ }^{\$ \$} p<0.001$ vs. control $P d g f r b b^{+/-}$mice, one-way ANOVA followed by Bonferroni's post-hoc test. n.s.: not significant.

PDGF-BB further enhanced the effect of PCM/PBS (PCM/ PBS, $p<0.001$; PCM/PDGF-BB, $p<0.001$, ANOVA; Fig. $4 F)$. Immunoblot analyses demonstrated that the phosphorylation of STAT3 and AKT, both crucial signaling molecules for the activation of astrocytes (Luo and Zheng, 2016), was significantly increased in astrocytes cultured with PCM/PBS (STAT3: $p=0.049$, AKT: $p=0.002$, ANOVA) and further increased with PCM/PDGF-BB (STAT3: $p=0.008$; AKT: $p=0.002$, ANOVA), while phosphorylation of ERK1/2 was not increased (Fig. 4G). Consistently, immunofluorescence staining demonstrated that STAT3 was strongly phosphorylated in GFAP- 
A

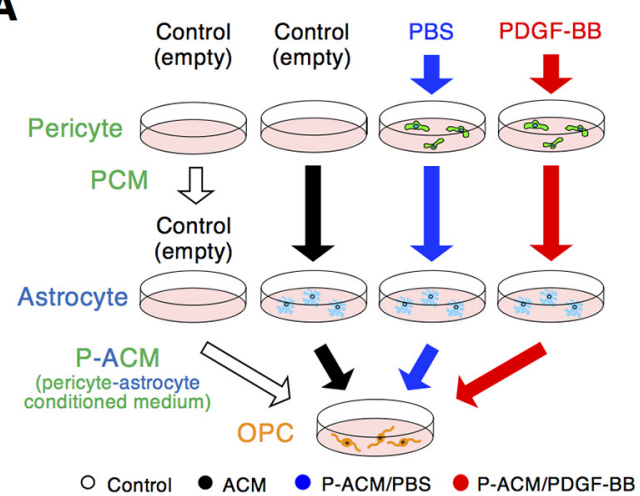

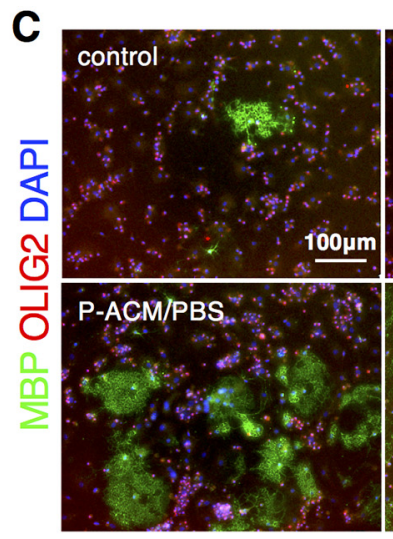

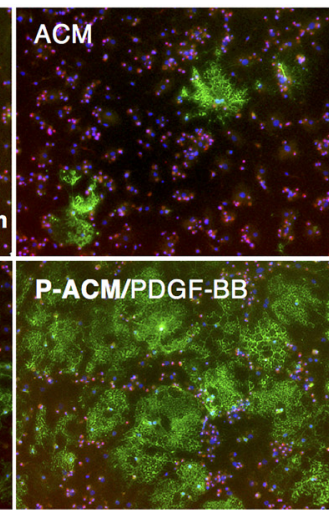

D
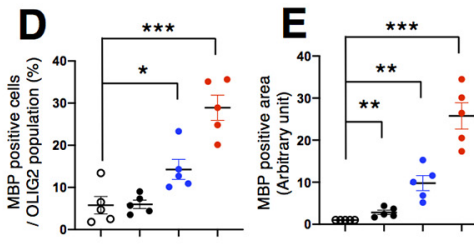

$\mathbf{F}$

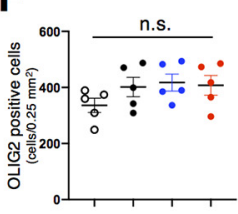

O Control

- P-ACM/PBS $\bullet$ P.ACMPDGF-BB
G

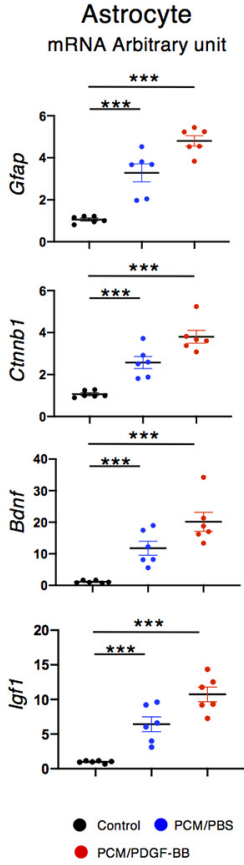

Figure 6. Astrocytes stimulated with PCM promote differentiation and myelination. $\boldsymbol{A}$, An experimental scheme for OPC differentiation in normal culture medium (control) or in astrocyte culture CM pretreated with empty pericyte medium, PCM/PBS, or PCM/ PDGF-BB. B, Quantitative PCR of Mbp, Mag, and Plp in OPCs stimulated with CM for $5 \mathrm{~d}(n=4$, each group). C, Double immunofluorescence labeling of MBP (green) and OLIG2 (red) in cultured OPCs treated with ACM for $7 \mathrm{~d}(n=5$, each group; scale bar, 100 $\mu \mathrm{m})$. $\boldsymbol{D}-\boldsymbol{F}$, Quantification of the number of MBP-positive cells among OLIG2-positive cells (D), MBP-positive areas (E), and OLIG2positive cells $(\boldsymbol{F})$. G, Quantitative PCR for Gfap, Ctnnb1, Bdnf, and Igf1 in astrocytes treated with PCM for $24 \mathrm{~h}$ ( $n=6$, each group). Data are shown as the mean \pm SEM. $\boldsymbol{B}, \boldsymbol{D}-\mathbf{G},{ }^{\dagger} p<0.1, * p<0.05, * * p<0.01$, and $* * * p<0.001$, one-way ANOVA followed by Bonferroni's post hoc test. n.s.: not significant.

positive astrocytes in peri-infarct areas after pMCAO, which was attenuated in Pdgfrb ${ }^{+/-}$mice $(p=0.041$, unpaired $t$ test; Fig. 4H). It is known that IL6 cytokine family proteins activate STAT3 and AKT in astrocytes (Yamashita et al., 2005; Gertz et al., 2012); therefore, we examined whether IL6 was expressed in pericytes in ischemic areas in vivo (Nakamura et al., 2012). Double immunofluorescence labeling demonstrated that IL6 was expressed in NG2-positive pericytes (Fig. 4/), as well as in F4/80-positive macrophages and around CD31-positve endothelial cells (Extended Data Fig. 42), within the infarct area. Furthermore, the mRNA level of $/ / 6$ was significantly increased in wild type on days 7 and 14 after pMCAO, compared with that of $P d g f r b^{+/-}$ mice (day $7, p=0.002$; day $14, p=0.009$, unpaired $t$ test; Fig. 4J). We confirmed that PDGF-BB increased the level of $/ 16$ mRNA in cultured pericytes $(p<0.001$, ANOVA), while pretreatment with SU16f abolished the PDGF-BB-mediated upregulation of $1 / 6$ ( $p<0.001$, ANOVA; Fig. 4K). Moreover, the growth effects of PCM on astrocytes were significantly attenuated by treatment with an anti-IL6 neutralizing antibody in a dosedependent manner (Fig. 4L). These findings suggested the presence of intercellular interaction between PDGFR $\beta$-positive pericytes and reactive astrocytes that is mediated, at least partly, through IL6.

\section{Peri-infarct oligodendrogenesis is attenuated in Pdgfrb $^{+/-}$mice}

We further examined whether PDGFR $\beta$-mediated repair could elicit neural reorganization other than astrogliosis in peri-infarct areas. We first examined the recruitment of DCX-positive immature neurons from the SVZ. Immunohistochemistry for DCX demonstrated that the recruitment of the immature neurons in the ipsilateral hemisphere was not significantly different between wild-type and $\mathrm{Pdgfrb}^{+/}$mice on days 14 and 28 after pMCAO (day 14, $p=0.302$; day 28, $p=0.766$, unpaired $t$ test; Fig. 5A,B). Furthermore, the number of EdU- and NeuN-double positive newly generated neurons in peri-infarct areas was not different on day 28 between the groups $\left(5 \pm 2\right.$ vs $5 \pm 3$ cells $/ \mathrm{mm}^{2}, p=0.886$; unpaired $t$ test; Fig. $5 C, D$ ). We then examined oligodendrogenesis in peri-infarct areas. The number of OLIG2-positive OPCs was significantly increased from day 7 to 28 equally in wild-type and $P$ dgfrb ${ }^{+/}$mice (day $7, p=0.272$; day $14, p=$ 0.897 ; day $28, p=0.489$, unpaired $t$ test; Fig. $5 E, F$ ). However, we found that the number of APC-positive differentiated OLs in peri-infarct areas was significantly greater in wild-type compared with $\mathrm{Pdgfrb}^{+/-}$mice on day $28\left(555 \pm 83\right.$ vs $391 \pm 52$ cells $/ \mathrm{mm}^{2}, p=0.004$, unpaired $t$ test; Fig. 5G,H). Furthermore, triple immunofluorescence staining demonstrated that the oligodendrogenesis and myelination, as shown by APC (green) and MBP (blue) 

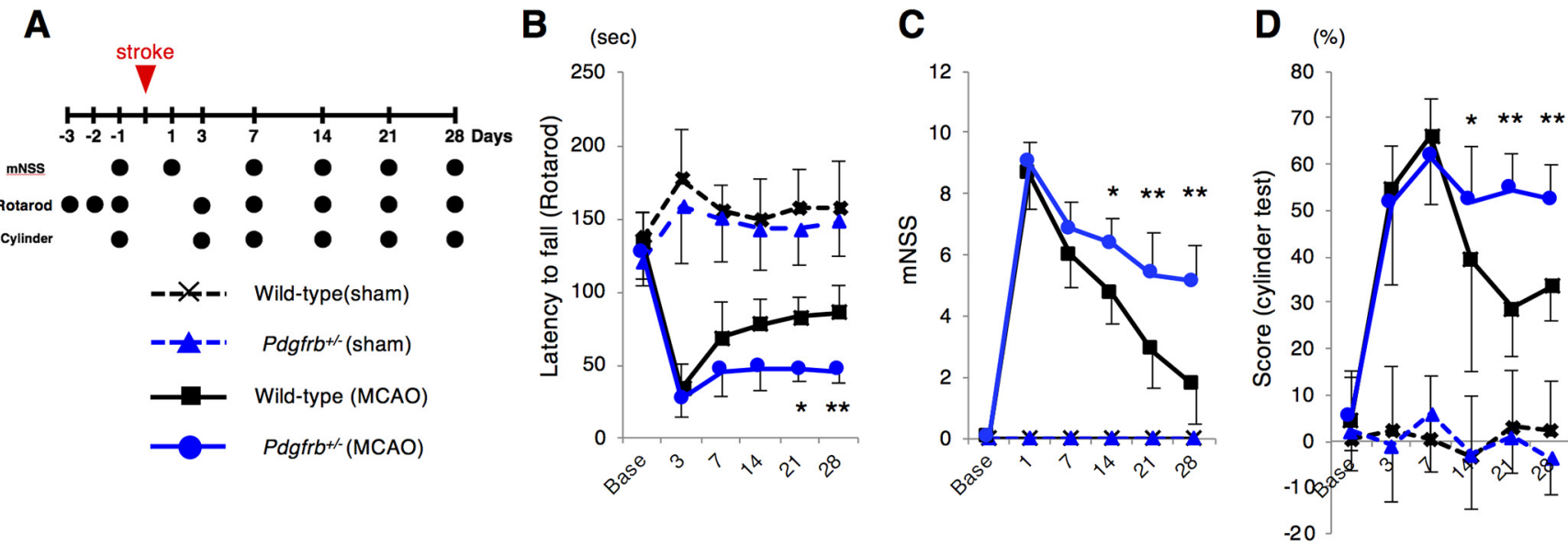

Figure 7. Functional recovery after pMCAO was attenuated in $\mathrm{Pdgfrb}^{+/}$mice. $\boldsymbol{A}$, Experimental schedule for neurological assessment. Neurological function was assessed by $(\boldsymbol{B})$ rotarod test, $(\boldsymbol{C}) \mathrm{mNSS}$ test, and $(\boldsymbol{D})$ cylinder test at baseline and days $1,3,7,14$, 21 , and 28 after pMCAO in wild-type (black) and Pdgfrb $^{+/-}$mice (blue). Data are shown as the mean \pm SEM $(n=8$, each group, $* p<0.05$, and $* * p<0.01$, unpaired $t$ test).

staining, occurred within GFAP (red)-positive astrogliosis in peri-infarct areas (Fig. 5/). Double immunofluorescence labeling with EdU and GST $\pi$ demonstrated that the number of newly generated OLs in peri-infarct areas was significantly greater on day 28 in wild-type than in Pdgfrb ${ }^{+/-}$ mice (34 \pm 9 vs $21 \pm 9$ cells $/ \mathrm{mm}^{2}, p=0.022$, unpaired $t$ test; Fig. $5 \mathrm{~J}, K)$. Moreover, the extent of axon myelination, as assessed by double immunofluorescence labeling with MBP and the pan-axonal neurofilament marker, SMI312, was significantly greater on day 28 in wild-type mice than in $\mathrm{Pdgfrb}^{+/-}$mice $(p=0.002$, unpaired $t$ test; Fig. $5 L, M)$. We confirmed that the extent of demyelination in the striatum and the corpus callosum on day 1 after pMCAO was not different between the groups (striatum, $p=0.394$; corpus callosum, $p=0.920$, unpaired $t$ test; Extended Data Fig. 5-1), indicating that post-stroke peri-infarct remyelination was significantly attenuated in $\mathrm{Pdgfrb}^{+/-}$mice.

\section{Astrocytes that are stimulated with PCM produce factors promoting differentiation and myelination of OPCs}

To elucidate how the differentiation of OPCs was induced in peri-infarct areas, we examined the intercellular interaction among pericytes, astrocytes, and OPCs, using cultured cells. We tested whether ACM, ACM treated with PCM/PBS (P-ACM/PBS), or ACM treated with PCM/ PDGF-BB (P-ACM/PDGF-BB) could promote the proliferation, differentiation and myelination of cultured OPCs (Fig. 6A). We examined expression of $M b p$, myelin associated glycoprotein (Mag), and myelin proteolipid protein $(P / p)$, markers of myelination, in OPCs by quantitative PCR. The largest increase in expression of these markers was seen after treatment with P-ACM/PDGF-BB, followed by PACM/PBS and then ACM (Mbp: ACM, $p=0.044$; P-ACM/ PBS, $p=0.026$; P-ACM/PDGF-BB, $p=0.002$; Mag: ACM, $p=0.002 ;$ P-ACM/PBS, $p=0.004 ;$ P-ACM/PDGF-BB, $p<$ 0.001 ; Plp: ACM, $p=0.069$; P-ACM/PBS, $p=0.013$; PACM/PDGF-BB, $p=0.005$, ANOVA; Fig. $6 B$ ). Consistently, double immunofluorescence labeling showed that the number of MBP-positive cells among OLIG2-positive cells (ACM, $p=0.930$; P-ACM/PBS, $p=0.028$; P-ACM/PDGF$\mathrm{BB}, p<0.001$, ANOVA; Fig. 6C,D) and in MBP-positive areas (ACM, $p=0.007$; P-ACM/PBS, $p=0.001$; P-ACM/ PDGF-BB, $p<0.001$, ANOVA; Fig. $6 C, E$ ) were greatest in OPCs treated with P-ACM/PDGF-BB, while the number of OLIG2-positive cells was not significantly different among the groups (Fig. 6C,F). We also examined the direct effects of PCM on OPCs. Although the numbers of MBP-positive cells among OLIG2-positive ones and MBP-positive areas were greater in OPCs treated with PCM/PBS and PCM/ PDGF-BB, their extents were much lower compared with those treated with ACM (Fig. 6; Extended Data Fig. 6-1). These findings indicated that astrocytes, stimulated with pericyte-derived molecules, may promote the differentiation and myelination of near-by OPCs.

We confirmed by quantitative PCR that PCM, particularly when conditioned with PDGF-BB, increased the expression of Gfap and Ctnnb1 ( $\beta$-catenin), markers of reactive astrocytes (Cregg et al., 2014; Hara et al., 2017), and of Bdnf and Igf1, trophic factors that promote OPC differentiation and myelination, in cultured astrocytes (Gfap: PCM/PBS, $p<0.001$; PCM/PDGF-BB, $p<0.001$ : Ctnnb1: PCM/PBS, $p<0.001$; PCM/PDGF-BB, $p<$ 0.001: Bdnf: PCM/PBS, $p<0.001$; PCM/PDGF-BB, $p<$ 0.001: Igf1: PCM/PBS, $p<0.001$; PCM/PDGF-BB, $p<0.001$, ANOVA; Fig. 6G).

\section{Functional recovery after pMCAO is significantly attenuated in Pdgfrb $^{+/-}$mice}

We finally tested whether the peri-infarct neural reorganization, including astrogliosis and oligodendrogenesis, enhanced by PDGFR $\beta$-mediated repair was associated with functional recovery after PMCAO, using wild-type and Pdgfrb $^{+/}$mice (Fig. 7A). Neurological functions, assessed by rotarod, mNSS, and cylinder tests, were decreased similarly in both mice on days 1-3; however, functional recovery after day 14 was significantly better in wild-type mice 

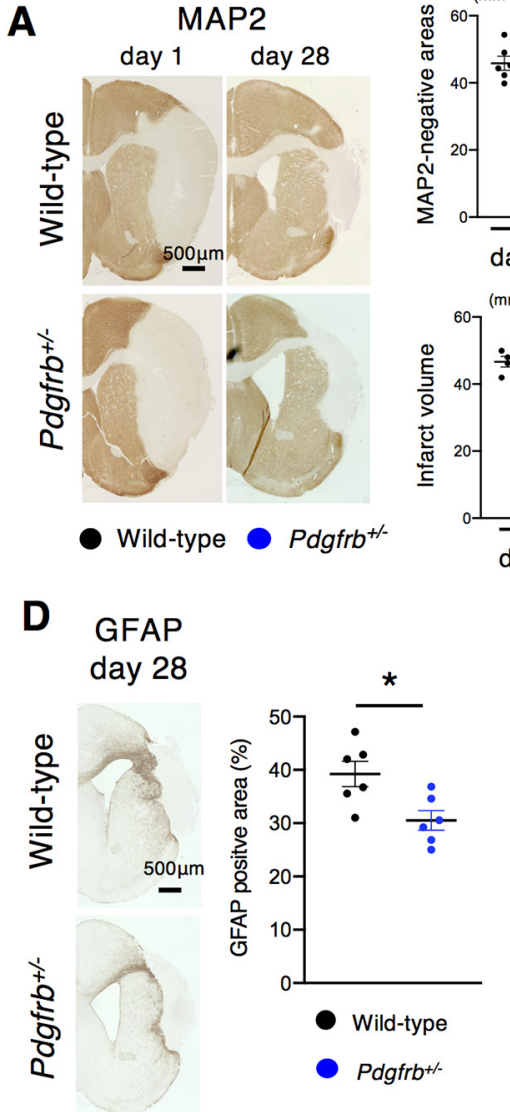

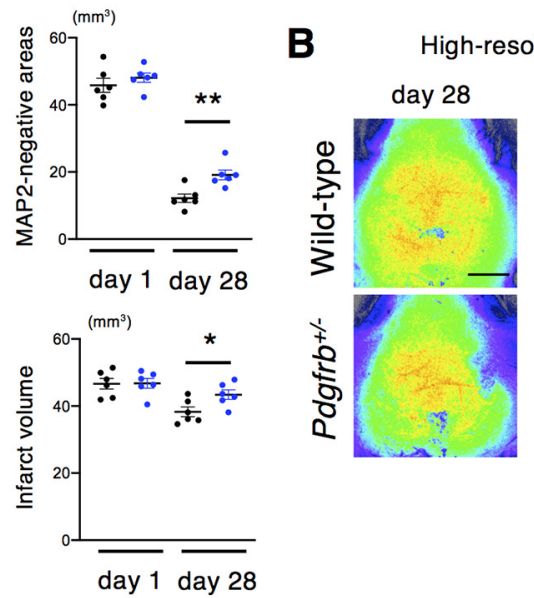

E

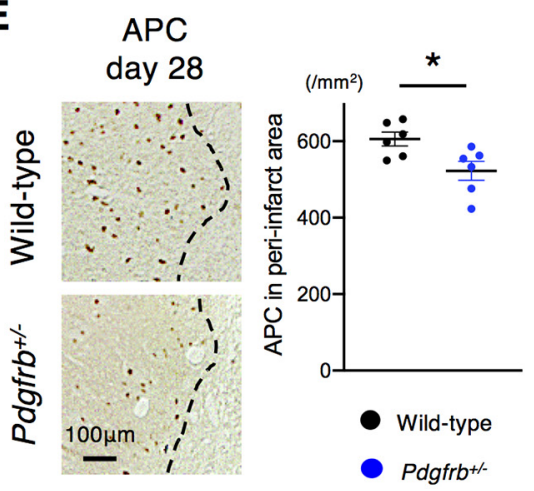

CD13

day 28

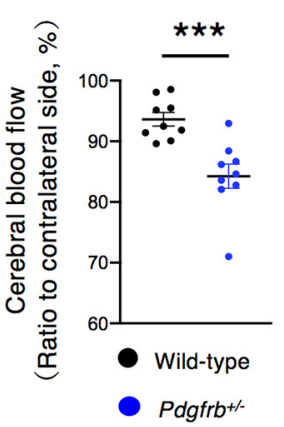

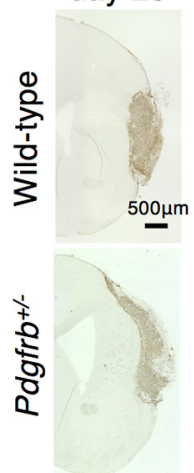

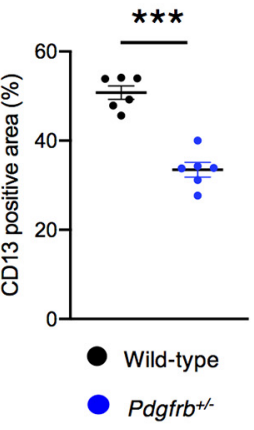

Figure 8. Post-stroke phenotypic changes in female Pdgfrb $^{+/}$mice. A, MAP2 staining on days 1 and 28 after pMCAO in female wild-type and Pdgfrb $^{+/-}$mice (scale bar, $500 \mu \mathrm{m}$ ). Quantification of MAP2-negative areas and infarct volume ( $n=6$, each group). Data are shown as the mean \pm SEM ( $* p<0.05$ and $* * p<0.01$, unpaired $t$ test). $\boldsymbol{B}$, Representative laser speckle images of CBF after pMCAO in female wild-type and Pdgfrb $^{+/-}$mice on day $28(n=9$, each group). Scale bar, $2 \mathrm{~mm}$. Data represent the mean \pm SEM (***p $<0.001$, unpaired $t$ test). $\boldsymbol{C}$, Representative CD13 staining (scale bar, $500 \mu \mathrm{m}$ ) and quantification on day 28 after pMCAO in female wild-type and Pdgfrb ${ }^{+/-}$mice $(n=6$, each group). Data are shown as the mean \pm SEM (***p $<0.001$, unpaired $t$ test). $\boldsymbol{D}$, Representative GFAP staining (scale bar, $500 \mu \mathrm{m}$ ) and quantification on day 28 after pMCAO in wild-type and Pdgfrb ${ }^{+/-}$ female mice ( $n=6$, each group). Data are shown as the mean \pm SEM ( $* p<0.05$, unpaired $t$ test). $E$, Representative APC staining (scale bar, $100 \mu \mathrm{m}$ ) and quantification on day 28 after pMCAO in female wild-type and Pdgfrb ${ }^{+/}$mice $(n=6$, each group). Data are shown as the mean \pm SEM $(* p<0.05$, unpaired $t$ test). $\boldsymbol{F}$, Neurological scores in female mice after pMCAO ( $n=9$, each group). Data represent the mean \pm SEM $\left({ }^{\dagger} p<0.1, * p<0.05\right.$, and $* * p<0.01$, unpaired $t$ test).

than in Pdgfrb ${ }^{+/-}$mice, consistent with the temporal profiles of intrainfarct repair and peri-infarct neural reorganization (Fig. 7B-D).

\section{Post-stroke phenotypic changes in female Pdgfrb $^{+/-}$ mice}

We also tested post-stroke phenotypic changes in female Pdgfrb ${ }^{+/-}$mice. The reduction of MAP2-negative areas and infarct volume was significantly attenuated (MAP2-negative areas, $p=0.004$; infarct volume, $p=$ 0.029 , unpaired $t$ test; Fig. $8 A$ ) with the recovery of CBF attenuated in ischemic areas $(p<0.001$, unpaired $t$ test; Fig. $8 B$ ) on day 28 after pMCAO. CD13-positive fibrotic formation was significantly attenuated ( $p<0.001$, unpaired $t$ test; Fig. 8C) accompanied with reduced GFAPpositive astrogliosis ( $p=0.016$, unpaired $t$ test; Fig. $8 D$ ) and APC-positive differentiated OLs $(p=0.023$, unpaired $t$ test; Fig. 8E) in peri-infarct areas on day 28. Consistently, post-stroke functional recovery was significantly impaired in female Pdgfrb ${ }^{+/-}$mice (Fig. 8F).

\section{Discussion}

We extensively examined the roles of pericytes, particularly PDGFR $\beta$-positive ones, after acute ischemic stroke, from tissue repair to functional recovery. First, the vascular mural cells expressing PDGFR $\beta$ enhances leptomeningeal arteriogenesis and intrainfarct angiogenesis, thereby promoting recovery of CBF in ischemic areas, which is a prerequisite for tissue repair (Tachibana et al., 2017; Gautam and Yao, 2018). Second, PDGFR $\beta$ mediated repair within infarct areas enhances peri-infarct astrogliosis and oligodendrogenesis, thereby promoting functional recovery (Fig. 9). To the best of our knowledge, this is the first report clearly demonstrating 


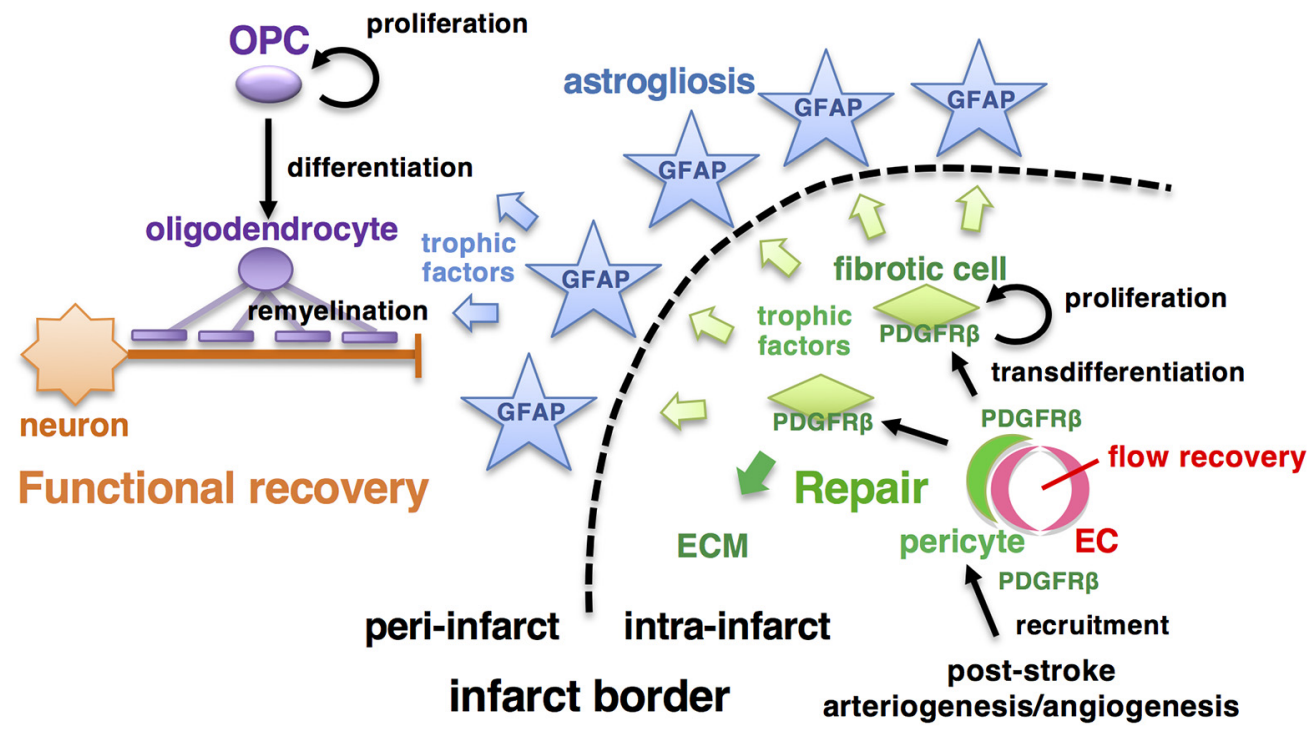

Figure 9. Schematic diagram of pericyte-mediated tissue repair and functional recovery after pMCAO. Schematic diagram of this study. PDGFR $\beta$ signaling in vascular mural cells plays important roles in mediating post-stroke leptomeningeal arteriogenesis and intrainfarct angiogenesis leading to the recovery of CBF in ischemic areas. After recruitment around endothelial cell (EC) tubes, PDGFR $\beta$-positive pericytes transdifferentiate into fibrotic cells, enhance tissue repair within infarct areas and astrogliosis in peri-infarct areas, and promote peri-infarct oligodendrogenesis (which together is OPC differentiation and myelination) leading to functional recovery. ECM, extracellular matrix.

that improved intrainfarct tissue repair may promote functional recovery through the enhancement of astrogliosis and oligodendrogenesis after acute ischemic stroke.

Some clinicians and researchers may consider that post-stroke recovery of CBF within infarct areas is unimportant because there are no neurons that can be rescued after brain infarction is complete. However, leptomeningeal arteriogenesis and intrainfarct angiogenesis certainly occurred (Figs. 2, 3) and contributed to recovery of CBF and subsequent fibrotic formation within infarct areas (Fig. 3). In response to PDGF-BB, PDGFR $\beta$-expressing pericytes/SMCs can produce cytokines and chemokines that recruit monocytes/macrophages. The monocytes/ macrophages recruited into the anastomotic vessels induced the vascular remodeling leading to vascular dilation (Scholz et al., 2000; Sugiyama et al., 2011). Because these processes were significantly attenuated in $\mathrm{Pdgfrb}^{+1}$ - mice (Fig. 2), PDGFR $\beta$ plays an important role in the formation of arteriogenesis in ischemic areas after pMCAO, a crucial factor for functional recovery (Komori et al., 2019). In addition, PDGFR $\beta$ plays crucial roles in microvascular pericytes. Pericytes are very vulnerable and easily detach from endothelial cells after ischemic insult (Tachibana et al., 2017), and thus they have to be re-recruited around endothelial tubes through PDGFR $\beta$ to stabilize microvascular structure and to maintain blood flow (Hellstrom et al., 1999; Daneman et al., 2010). PDGFR $\beta$ expressing pericytes transdifferentiate into fibrotic cells and occupy infarct areas to complete the repair while producing extracellular matrix proteins (Makihara et al., 2015). Collectively, pericytes and SMCs may play crucial roles in arteriogenesis, angiogenesis and fibrosis after ischemic stroke through PDGFR $\beta$.
It has been controversial whether the fibrotic tissue repair within injured areas in the CNS is beneficial or not, because it may hinder the chance of neurogenesis and axonal regeneration, particularly after spinal cord injury (Dias and Göritz, 2018; Gautam and Yao, 2018). However, the present study clearly demonstrated that PDGFR $\beta$ mediated fibrotic repair correlated with better functional recovery during the subacute phase after ischemic stroke (Fig. 7). The benefits of postinjury fibrotic repair may be different between the brain and the spinal cord or be dependent upon the timing and/or extent of tissue damage (Adams and Gallo, 2018). A possible mechanism underlying the positive association between intrainfarct repair and functional recovery may be that $\operatorname{PDGFR} \beta$-positive pericytes or pericyte-derived cells have gene expression profiles similar to those of mesenchymal stem cells and create a regenerative microenvironment in intrainfarct and peri-infarct areas in the brain while producing various neurotrophic factors, such as BDNF and NT3, and immunomodulatory molecules (Ishitsuka et al., 2012; Chen et al., 2014; Caplan, 2017).

In the present study, defective intrainfarct repair by genetic manipulation of Pdgfrb significantly attenuated peri-infarct astrogliosis and oligodendrogenesis, but not neurogenesis. As reported previously, there may be a close association between PDGFR $\beta$-expressing cells and GFAP-positive reactive astrocytes through direct contact with one another at the boundary of brain infarction (Shen et al., 2012; Wanner et al., 2013; Makihara et al., 2015). Based on the present results, we consider that intrainfarct PDGFR $\beta$-positive cells positively regulate astrocyte activity by producing humoral factors (Fig. 4). Among possible factors produced by PDGFR $\beta$-positive cells, we focused on IL6 because it can be produced from 
mesenchymal cells, including pericytes (Gaceb et al., 2018), and can induce the phosphorylation of STAT3 and AKT, both potent intracellular transducers of activation and survival/proliferation of astrocytes (Yamashita et al., 2005; Nakamura et al., 2012; Wanner et al., 2013; Gu et al., 2016). It is also reported that IL6 can induce poststroke angiogenesis and repair (Gertz et al., 2012). Indeed, local production of IL6 by pericytes was significantly increased on 7 and $14 \mathrm{~d}$ after pMCAO and was significantly attenuated in $\mathrm{Pdgfrb}^{+/}$mice. Furthermore, PDGF-BB increased the production of IL6 in cultured PDGFR $\beta$-positive pericytes, while PCM-induced proliferation of cultured astrocytes was attenuated by treatment with an anti-IL6 antibody. Thus, humoral factors produced by PDGFR $\beta$-positive cells, including IL6, may promote astrocyte activation and survival/proliferation in peri-infarct areas (Zamanian et al., 2012).

The most important and interesting finding of this study is that $\operatorname{PDGFR} \beta$-mediated intrainfarct repair enhanced peri-infarct oligodendrogenesis as well as astrogliosis (Fig. 5). Oligodendrogenesis consists of three main steps, OPC proliferation, OPC differentiation and myelination (Franklin and Ffrench-Constant, 2008; Marin and Carmichael, 2019). Post-stroke OPC proliferation, as assessed by OLIG2-positive cell number, was promptly induced in peri-infarct areas within $7 \mathrm{~d}$, regardless of intrainfarct tissue repair (Fig. 5). Although we could not identify the specific factors promoting the proliferation of OPCs, demyelination or ischemic insult in the brain may stimulate the proliferation (Franklin and Ffrench-Constant, 2008; Itoh et al., 2015; Marin and Carmichael, 2019). However, the subsequent OPC differentiation and myelination may require significant levels of nutrients and/or trophic factors in an appropriate environment, probably astrogliosis (Burda and Sofroniew, 2014; Cregg et al., 2014; Miyamoto et al., 2015). Consistent with this in vivo observation, ACM could induce the differentiation of OPCs and the production of myelinating proteins by cultured OPCs (Fig. 6). Because these responses were enhanced further by the addition of PDGF-BB-stimulated PCM to ACM (Fig. 6), we assume that peri-infarct reactive astrocytes, which are activated by PDGFR $\beta$-expressing cells within infarct areas, are primarily responsible for the OPC differentiation and remyelination. However, we could not exclude the possibility that molecules produced from PDGFR $\beta$-expressing cells directly stimulate OPC differentiation without astrocyte responses, as shown in Extended Data Figure 6-1 and in non-stroke demyelinating animal models (De La Fuente et al., 2017; Montagne et al., 2018). The post-stroke intercellular interaction among pericytes-astrocytes-OLs/OPCs may be essential to complete efficient remyelination in the brain. This may be quite similar to the developmental myelination process: astrogenesis and oligodendrogenesis occur sequentially after the recruitment of PDGFR $\beta$-positive pericytes into endothelial tubes (Daneman et al., 2010). We believe that the concept of repair-induced OPC differentiation and remyelination is very important and a feasible therapeutic strategy for promoting functional recovery after acute ischemic stroke.
There are some limitations in the present study. First, we used conventional $P d g \mathrm{frb}^{+/-}$mice. PDGFR $\beta$ is exclusively expressed in pericytes in mice, particularly after ischemic stroke (Bell et al., 2010; Winkler et al., 2010; Arimura et al., 2012; Makihara et al., 2015); therefore, we considered that the effects of PDGFR $\beta$ heterozygous deletion after ischemic stroke were attributable mainly to impaired function of pericytes. However, we cannot completely exclude the possibility that PDGFR $\beta$ might be expressed in other cell types, such as neurons, astrocytes, and OPCs, and play a role in post-stroke neurorestoration (Shen et al., 2012). Second, because we used the mice with systemic deletion of Pdgfrb, there remains a possibility that PDGFR $\beta$-expressing cells in non-CNS organs produced some trophic factors in response to stroke, thereby affecting post-stroke recovery as remote effects. The finding that post-stroke body weight loss was greater in $P$ dgfrb ${ }^{+/-}$mice (Figs. 1-3) may be one of the systemic effects of heterozygous deletion of Pdgfrb. Third, we may need to consider other cell types, such as endothelial cells (Zhou et al., 2019), macrophages (Cantuti-Castelvetri et al., 2018) and regulatory T cells (Dombrowski et al., 2017) for their contribution to enhanced oligodendrogenesis after ischemic stroke, as has been shown in demyelinating disorders, although there is a possibility that these cells function cooperatively with pericytes/SMCs and astrocytes to promote oligodendrogenesis. Fourth, although PDGFR $\beta$-mediated intrainfarct repair did not directly affect the number of newly generated neurons in peri-infarct areas (Fig. 5), the possibility remains that it might enhance axonal sprouting of survived or migrating neurons to form new inter-neuron connections in peri-infarct areas, thereby promoting functional recovery (Carmichael et al., 2017). Even so, remyelination is absolutely needed for these neurons to be functional. Fifth, Su et al. (2008) showed that simultaneous administration of imatinib, a PDGFR inhibitor, and recombinant tissue plasminogen activator reduces vascular permeability and hemorrhagic complications in an ischemia-reperfusion animal model (Su et al., 2008). We should note that the significance of PDGFR $\beta$ expression may be different between PMCAO and ischemia/reperfusion tMCAO models because post-stroke development of leptomeningeal arteriogenesis and intrainfarct angiogenesis are not always necessary in ischemia-reperfusion stroke models in contrast to pMCAO (Tachibana et al., 2017; Komori et al., 2019). In addition, Su et al. (2008) only evaluated cerebrovascular permeability and hemorrhage $1 \mathrm{~d}$ after reperfusion, but not functional recovery in the subacute phase (Su et al., 2008). Finally, although we demonstrated the importance of intercellular interaction among pericytes, astrocytes, and OPCs/OLs in tissue repair and functional recovery, we could not identify the decisive factors responsible for the interaction in this study. We should identify the factors mediating the interaction that leads to functional recovery in future.

In conclusion, we demonstrate that improved tissue repair within infarct areas can bring about better functional recovery through the enhancement of astrogliosis and oligodendrogenesis during the subacute phase. We 
speculate that post-stroke oligodendrogenesis leading to functional recovery can occur in an appropriate environment, with sufficient blood supply and the presence of active astrocytes. We suggest that promotion of repair is a promising therapeutic regenerative medicine strategy after acute ischemic stroke even considering the limited therapeutic time window.

\section{References}

Adams KL, Gallo V (2018) The diversity and disparity of the glial scar. Nat Neurosci 21:9-15.

Anderson MA, Burda JE, Ren Y, Ao Y, O'Shea TM, Kawaguchi R, Coppola G, Khakh BS, Deming TJ, Sofroniew MV (2016) Astrocyte scar formation aids central nervous system axon regeneration. Nature 532:195-200.

Arimura K, Ago T, Kamouchi M, Nakamura K, Ishitsuka K, Kuroda J, Sugimori H, Ooboshi H, Sasaki T, Kitazono T (2012) PDGF receptor $\beta$ signaling in pericytes following ischemic brain injury. Curr Neurovasc Res 9:1-9.

Armulik A, Abramsson A, Betsholtz C (2005) Endothelial/pericyte interactions. Circ Res 97:512-523.

Armulik A, Genové G, Betsholtz C (2011) Pericytes: Developmental, physiological, and pathological perspectives, problems, and promises. Dev Cell 21:193-215.

Ayata C, Dunn AK, Gursoy OY, Huang Z, Boas DA, Moskowitz MA (2004) Laser speckle flowmetry for the study of cerebrovascular physiology in normal and ischemic mouse cortex. J Cereb Blood Flow Metab 24:744-755.

Bell RD, Winkler EA, Sagare AP, Singh I, LaRue B, Deane R, Zlokovic BV (2010) Pericytes control key neurovascular functions and neuronal phenotype in the adult brain and during brain aging. Neuron 68:409-427.

Burda JE, Sofroniew MV (2014) Reactive gliosis and the multicellular response to CNS damage and disease. Neuron 81:229-248.

Cantuti-Castelvetri L, Fitzner D, Bosch-Queralt M, Weil MT, Su M, Sen P, Ruhwedel T, Mitkovski M, Trendelenburg G, Lütjohann D, Möbius W, Simons M (2018) Defective cholesterol clearance limits remyelination in the aged central nervous system. Science 359:684-688.

Caplan Al (2017) New MSC: MSCs as pericytes are sentinels and gatekeepers. J Orthop Res 35:1151-1159.

Carmichael ST, Kathirvelu B, Schweppe CA, Nie EH (2017) Molecular, cellular and functional events in axonal sprouting after stroke. Exp Neurol 287:384-394.

Chen J, Venkat P, Zacharek A, Chopp M (2014) Neurorestorative therapy for stroke. Front Hum Neurosci 8:382.

Cramer SC (2008) Repairing the human brain after stroke: I. Mechanisms of spontaneous recovery. Ann Neurol 63:272-287.

Cregg JM, DePaul MA, Filous AR, Lang BT, Tran A, Silver J (2014) Functional regeneration beyond the glial scar. Exp Neurol 253:197-207.

Daneman R, Zhou L, Kebede AA, Barres BA (2010) Pericytes are required for blood-brain barrier integrity during embryogenesis. Nature 468:562-566.

De La Fuente AG, Lange S, Silva ME, Gonzalez GA, Tempfer H, van Wijngaarden $P$, Zhao C, Di Canio L, Trost A, Bieler L, Zaunmair P, Rotheneichner P, O'Sullivan A, Couillard-Despres S, Errea O, Mäe MA, Andrae J, He L, Keller A, Bátiz LF, et al. (2017) Pericytes stimulate oligodendrocyte progenitor cell differentiation during CNS remyelination. Cell Rep 20:1755-1764.

Dias DO, Göritz C (2018) Fibrotic scarring following lesions to the central nervous system. Matrix Biol 68-69:561-570.

Dias DO, Kim H, Holl D, Werne Solnestam B, Lundeberg J, Carlén M, Göritz C, Frisén J (2018) Reducing pericyte-derived scarring promotes recovery after spinal cord injury. Cell 173:153-165.e22.

Dombrowski Y, O'Hagan T, Dittmer M, Penalva R, Mayoral SR, Bankhead P, Fleville S, Eleftheriadis G, Zhao C, Naughton M,
Hassan R, Moffat J, Falconer J, Boyd A, Hamilton P, Allen IV, Kissenpfennig A, Moynagh PN, Evergren E, Perbal B, et al. (2017) Regulatory $T$ cells promote myelin regeneration in the central nervous system. Nat Neurosci 20:674-680.

Faiz M, Sachewsky N, Gascón S, Bang KW, Morshead CM, Nagy A (2015) Adult neural stem cells from the subventricular zone give rise to reactive astrocytes in the cortex after stroke. Cell Stem Cell 17:624-634.

Franklin RJ, Ffrench-Constant C (2008) Remyelination in the CNS: From biology to therapy. Nat Rev Neurosci 9:839-855.

Gaceb A, Özen I, Padel T, Barbariga M, Paul G (2018) Pericytes secrete pro-regenerative molecules in response to platelet-derived growth factor-BB. J Cereb Blood Flow Metab 38:45-57.

Gautam J, Yao Y (2018) Roles of pericytes in stroke pathogenesis. Cell Transplant 27:1798-1808.

Gertz K, Kronenberg G, Kälin RE, Baldinger T, Werner C, Balkaya M, Eom GD, Hellmann-Regen J, Kröber J, Miller KR, Lindauer U, Laufs U, Dirnagl U, Heppner FL, Endres M (2012) Essential role of interleukin-6 in post-stroke angiogenesis. Brain 135:1964-1980.

Ginsberg MD (2008) Neuroprotection for ischemic stroke: Past, present and future. Neuropharmacology 55:363-389.

Göritz C, Dias DO, Tomilin N, Barbacid M, Shupliakov O, Frisén J (2011) A pericyte origin of spinal cord scar tissue. Science 333:238-242.

Goyal M, Menon BK, van Zwam WH, Dippel DW, Mitchell PJ, Demchuk AM, Dávalos $A$, Majoie $C B$, van der Lugt $A$, de Miquel MA, Donnan GA, Roos YB, Bonafe A, Jahan R, Diener HC, van den Berg LA, Levy El, Berkhemer OA, Pereira VM, Rempel J, et al. (2016) Endovascular thrombectomy after large-vessel ischaemic stroke: A meta-analysis of individual patient data from five randomised trials. Lancet 387:1723-1731.

Gu Y, He M, Zhou X, Liu J, Hou N, Bin T, Zhang Y, Li T, Chen J (2016) Endogenous IL-6 of mesenchymal stem cell improves behavioral outcome of hypoxic-ischemic brain damage neonatal rats by supressing apoptosis in astrocyte. Sci Rep 6:18587.

Hara M, Kobayakawa K, Ohkawa Y, Kumamaru H, Yokota K, Saito T, Kijima K, Yoshizaki S, Harimaya K, Nakashima Y, Okada S (2017) Interaction of reactive astrocytes with type I collagen induces astrocytic scar formation through the integrin- $\mathrm{N}$-cadherin pathway after spinal cord injury. Nat Med 23:818-828.

Hellstrom M, Kalén M, Lindahl P, Abramsson A, Betsholtz C (1999) Role of PDGF-B and PDGFR-beta in recruitment of vascular smooth muscle cells and pericytes during embryonic blood vessel formation in the mouse. Development 126:3047-3055.

Ishitsuka K, Ago T, Arimura K, Nakamura K, Tokami H, Makihara N, Kuroda J, Kamouchi M, Kitazono T (2012) Neurotrophin production in brain pericytes during hypoxia: A role of pericytes for neuroprotection. Microvasc Res 83:352-359.

Itoh K, Maki T, Lok J, Arai K (2015) Mechanisms of cell-cell interaction in oligodendrogenesis and remyelination after stroke. Brain Res 1623:135-149.

Iwai M, Stetler RA, Xing J, Hu X, Gao Y, Zhang W, Chen J, Cao G (2010) Enhanced oligodendrogenesis and recovery of neurological function by erythropoietin after neonatal hypoxic/ischemic brain injury. Stroke 41:1032-1037.

Komori M, Ago T, Wakisaka Y, Nakamura K, Tachibana M, Yoshikawa Y, Shibahara T, Yamanaka K, Kuroda J, Kitazono T (2019) Early initiation of a factor Xa inhibitor can attenuate tissue repair and neurorestoration after middle cerebral artery occlusion. Brain Res 1718:201-211.

Levine JM, Reynolds R, Fawcett JW (2001) The oligodendrocyte precursor cell in health and disease. Trends Neurosci 24:39-47.

Lo EH, Dalkara T, Moskowitz MA (2003) Mechanisms, challenges and opportunities in stroke. Nat Rev Neurosci 4:399-415.

Luo Y, Zheng SG (2016) Hall of fame among pro-inflammatory cytokines: Interleukin-6 gene and its transcriptional regulation mechanisms. Front Immunol 7:604.

Makihara N, Arimura K, Ago T, Tachibana M, Nishimura A, Nakamura K, Matsuo R, Wakisaka Y, Kuroda J, Sugimori H, Kamouchi M, Kitazono T (2015) Involvement of platelet-derived growth factor 
receptor $\beta$ in fibrosis through extracellular matrix protein production after ischemic stroke. Exp Neurol 264:127-134.

Marin MA, Carmichael ST (2019) Mechanisms of demyelination and remyelination in the young and aged brain following white matter stroke. Neurobiol Dis 126:5-12.

Matsuda T, Irie T, Katsurabayashi S, Hayashi Y, Nagai T, Hamazaki $\mathrm{N}$, Adefuin AMD, Miura F, Ito T, Kimura H, Shirahige K, Takeda T, Iwasaki K, Imamura T, Nakashima K (2019) Pioneer factor NeuroD1 rearranges transcriptional and epigenetic profiles to execute microglia-neuron conversion. Neuron 101:472-485.e7.

Miyamoto N, Maki T, Shindo A, Liang AC, Maeda M, Egawa N, Itoh K, Lo EK, Lok J, Ihara M, Arai K (2015) Astrocytes promote oligodendrogenesis after white matter damage via brain-derived neurotrophic factor. J Neurosci 35:14002-14008.

Montagne A, Nikolakopoulou AM, Zhao Z, Sagare AP, Si G, Lazic D, Barnes SR, Daianu M, Ramanathan A, Go A, Lawson EJ, Wang Y, Mack WJ, Thompson PM, Schneider JA, Varkey J, Langen R, Mullins E, Jacobs RE, Zlokovic BV (2018) Pericyte degeneration causes white matter dysfunction in the mouse central nervous system. Nat Med 24:326-337.

Nakamura K, Kamouchi M, Arimura K, Nishimura A, Kuroda J, Ishitsuka K, Tokami H, Sugimori H, Ago T, Kitazono T (2012) Extracellular acidification activates cAMP responsive element binding protein via $\mathrm{Na}+/ \mathrm{H}+$ exchanger isoform 1-mediated $\mathrm{Ca}^{2+}$ oscillation in central nervous system pericytes. Arterioscler Thromb Vasc Biol 32:2670-2677.

National Institute of Neurological D and Stroke rt-PA Stroke Study Group (1995) Tissue plasminogen activator for acute ischemic stroke. N Engl J Med 333:1581-1587.

Ohab JJ, Fleming S, Blesch A, Carmichael ST (2006) A neurovascular niche for neurogenesis after stroke. J Neurosci 26:13007-13016.

Okyere B, Creasey M, Lebovitz Y, Theus MH (2018) Temporal remodeling of pial collaterals and functional deficits in a murine model of ischemic stroke. J Neurosci Methods 293:86-96.

Rosenzweig S, Carmichael ST (2015) The axon-glia unit in white matter stroke: Mechanisms of damage and recovery. Brain Res 1623:123-134.

Scholz D, Ito W, Fleming I, Deindl E, Sauer A, Wiesnet M, Busse R, Schaper J, Schaper W (2000) Ultrastructure and molecular histology of rabbit hind-limb collateral artery growth (arteriogenesis). Virchows Arch 436:257-270.

Shen J, Ishii Y, Xu G, Dang TC, Hamashima T, Matsushima T, Yamamoto S, Hattori Y, Takatsuru Y, Nabekura J, Sasahara M (2012) PDGFR- $\beta$ as a positive regulator of tissue repair in a mouse model of focal cerebral ischemia. J Cereb Blood Flow Metab 32:353-367.

Shi Y, Jiang X, Zhang L, Pu H, Hu X, Zhang W, Cai W, Gao Y, Leak RK, Keep RF, Bennett MV, Chen J (2017) Endothelium-targeted overexpression of heat shock protein 27 ameliorates blood-brain barrier disruption after ischemic brain injury. Proc Natl Acad Sci USA 114:E1243-E1252.
Soriano P (1994) Abnormal kidney development and hematological disorders in PDGF beta-receptor mutant mice. Genes Dev 8:18881896.

Su EJ, Fredriksson L, Geyer M, Folestad E, Cale J, Andrae J, Gao Y, Pietras K, Mann K, Yepes M, Strickland DK, Betsholtz C, Eriksson U, Lawrence DA (2008) Activation of PDGF-CC by tissue plasminogen activator impairs blood-brain barrier integrity during ischemic stroke. Nat Med 14:731-737.

Sugiyama Y, Yagita Y, Oyama N, Terasaki Y, Omura-Matsuoka E, Sasaki T, Kitagawa K (2011) Granulocyte colony-stimulating factor enhances arteriogenesis and ameliorates cerebral damage in a mouse model of ischemic stroke. Stroke 42:770-775.

Sweeney MD, Ayyadurai S, Zlokovic BV (2016) Pericytes of the neurovascular unit: Key functions and signaling pathways. Nat Neurosci 19:771-783.

Tachibana M, Ago T, Wakisaka Y, Kuroda J, Shijo M, Yoshikawa Y, Komori M, Nishimura A, Makihara N, Nakamura K, Kitazono T (2017) Early reperfusion after brain ischemia has beneficial effects beyond rescuing neurons. Stroke 48:2222-2230.

Tognatta R, Miller RH (2016) Contribution of the oligodendrocyte lineage to CNS repair and neurodegenerative pathologies. Neuropharmacology 110:539-547.

Wanner IB, Anderson MA, Song B, Levine J, Fernandez A, GrayThompson Z, Ao Y, Sofroniew MV (2013) Glial scar borders are formed by newly proliferated, elongated astrocytes that interact to corral inflammatory and fibrotic cells via STAT3-dependent mechanisms after spinal cord injury. J Neurosci 33:12870-12886.

Winkler EA, Bell RD, Zlokovic BV (2010) Pericyte-specific expression of PDGF beta receptor in mouse models with normal and deficient PDGF beta receptor signaling. Mol Neurodegener 5:32.

Xing C, Hayakawa K, Lo EH (2017) Mechanisms, imaging, and therapy in stroke recovery. Transl Stroke Res 8:1-2.

Yamashita T, Sawamoto K, Suzuki S, Suzuki N, Adachi K, Kawase T, Mihara M, Ohsugi Y, Abe K, Okano H (2005) Blockade of interleukin-6 signaling aggravates ischemic cerebral damage in mice: Possible involvement of Stat3 activation in the protection of neurons. J Neurochem 94:459-468.

Yukami T, Yagita Y, Sugiyama Y, Oyama N, Watanabe A, Sasaki T, Sakaguchi M, Mochizuki H, Kitagawa K (2015) Chronic elevation of tumor necrosis factor- $\alpha$ mediates the impairment of leptomeningeal arteriogenesis in db/db mice. Stroke 46:1657-1663.

Zamanian JL, Xu L, Foo LC, Nouri N, Zhou L, Giffard RG, Barres BA (2012) Genomic analysis of reactive astrogliosis. J Neurosci 32:6391-6410.

Zhang R, Chopp M, Zhang ZG (2013) Oligodendrogenesis after cerebral ischemia. Front Cell Neurosci 7:201.

Zhou T, Zheng Y, Sun L, Badea SR, Jin Y, Liu Y, Rolfe AJ, Sun H, Wang $X$, Cheng Z, Huang Z, Zhao N, Sun X, Li J, Fan J, Lee C, Megraw TL, Wu W, Wang G, Ren Y (2019) Microvascular endothelial cells engulf myelin debris and promote macrophage recruitment and fibrosis after neural injury. Nat Neurosci 22:421-435. 